\title{
SARS-CoV-2 RNA quantification using droplet digital RT-PCR
}

Natalie N. Kinloch ${ }^{1,2}$, Gordon Ritchie ${ }^{3,4}$, Winnie Dong ${ }^{2}$, Kyle D. Cobarrubias ${ }^{2}$, Hanwei

Sudderuddin $^{2}$, Tanya Lawson ${ }^{3}$, Nancy Matic ${ }^{3,4}$, Julio S.G. Montaner, ${ }^{2,5}$, Victor Leung 3 ,4,5, Marc G. Romney ${ }^{3,4}$, Christopher F. Lowe $e^{3,4}$, Chanson J. Brumme ${ }^{2,5 \#}$, Zabrina L. Brumme e $^{1,2 \#}$

\#denotes equal contribution

${ }^{1}$ Faculty of Health Sciences, Simon Fraser University, Burnaby, British Columbia, Canada

${ }^{2}$ British Columbia Centre for Excellence in HIV/AIDS, Vancouver, British Columbia, Canada ${ }^{3}$ Division of Medical Microbiology and Virology, St Paul's Hospital, Vancouver, British Columbia, Canada

${ }^{4}$ Department of Pathology and Laboratory Medicine, University of British Columbia, Vancouver, British Columbia, Canada

${ }^{5}$ Department of Medicine, University of British Columbia, Vancouver, British Columbia, Canada

Running Title: SARS-CoV-2 RNA quantification using RT-ddPCR

Keywords: SARS-CoV-2; RT-ddPCR; ddPCR; viral load; quantification; COVID-19

Correspondent Footnote:

Zabrina L. Brumme, Ph.D.

Professor, Faculty of Health Sciences

Simon Fraser University

8888 University Drive

Burnaby, BC, Canada

V5A $1 \mathrm{~S} 6$

778-782-8872

zbrumme@sfu.ca

Chanson J. Brumme, Ph.D.

Assistant Professor, Faculty of Medicine

University of British Columbia

675-1081 Burrard Street, St. Paul's Hospital

Vancouver, BC

V6Z 1 Y 6

604-682-2344 ext. 63211

cbrumme@bccfe.ca 


\section{Abstract}

45

46

47

48

49

50

51

52

53

54

55

64 log-linear relationship can be used to mathematically derive SARS-CoV-2 RNA copy numbers

65 from $\mathrm{C}_{\mathrm{t}}$ values, allowing the wealth of available diagnostic test data to be harnessed to address

66 foundational questions in SARS-CoV-2 biology. 


\section{Introduction}

Quantitative viral load assays have revolutionized our ability to manage viral diseases (1-

72 6). While not yet widely available for SARS-CoV-2, quantitative assays could advance our

73 understanding of COVID-19 biology and inform infection prevention and control measures (7,

74 8). Most SARS-CoV-2 molecular diagnostic assays however, which use real-time reverse

75 transcriptase PCR (RT-PCR) to detect one or more SARS-CoV-2 genomic targets using

76 sequence-specific primers coupled with a fluorescent probe, are only semi-quantitative. These

77 tests produce cycle threshold $\left(\mathrm{C}_{\mathrm{t}}\right)$ values as readouts, which represent the PCR cycle where the

78 sample began to produce fluorescent signal above background. While each $\mathrm{C}_{\mathrm{t}}$ value decrement

79 corresponds to a roughly two-fold higher viral load (due to the exponential nature of PCR

80 amplification), $\mathrm{C}_{\mathrm{t}}$ values cannot be directly interpreted as SARS-CoV-2 viral loads without

81 calibration to a quantitative standard (9). Rather, $C_{t}$ values are interpreted as positive,

82 indeterminate or negative based on assay-specific cutoffs and evolving clinical guidelines. Due

83 to differences in nucleic acid extraction method, viral target and other parameters, $\mathrm{C}_{\mathrm{t}}$ values are

84 also not directly comparable across assays or technology platforms.

85 Reverse transcriptase droplet digital PCR (RT-ddPCR) offers an attractive platform for

86 SARS-CoV-2 RNA quantification $(10,11)$. Like real-time RT-PCR, ddPCR employs target-

87 specific primers coupled with a fluorescent probe, making it relatively straightforward to adapt

88 assays. In ddPCR however, each reaction is fractionated into 20,000 nanolitre-sized droplets

89 prior to massively parallel PCR amplification. At end-point, each droplet is categorized as

90 positive (target present) or negative (target absent), allowing for absolute target quantification

91 using Poisson statistics. This sensitive and versatile technology has been used for mutation

92 detection and copy number determination in the human genome (12), target verification 
93 following genome editing (13), and copy number quantification for viral pathogens (14-19).

94 Several real-time RT-PCR SARS-CoV-2-specific primer/probe sets have been used in RT-

$95 \operatorname{ddPCR}(10,11,20-22)$ with results achieving high sensitivity in some reports $(11,21,23-25)$, but

96 few studies have rigorously evaluated SARS-CoV-2-specific primer/probe set performance in

97 RT-ddPCR using RNA as a template. Furthermore, no studies to our knowledge have calibrated

98 SARS-CoV-2 viral loads to diagnostic test $\mathrm{C}_{\mathrm{t}}$ values. Here, we evaluate eight SARS-CoV-2-

99 specific primer/probe sets originally developed for real-time RT-PCR (26), for use in RT-

100 ddPCR. We also derive a linear equation relating RT-ddPCR-derived SARS-CoV-2 viral loads

101 and real-time RT-PCR-derived $C_{t}$ values for a commercial diagnostic assay, the LightMix ${ }^{\circledR}$

102 Modular SARS-CoV (COVID19) E-gene assay, allowing conversion of existing COVID-19

103 diagnostic results to viral loads. 


\section{Materials and Methods}

Primer and Probe Sets

10819 diagnostic assays (26) were assessed for use in RT-ddPCR (Table 1). These included the

109 Charité-Berlin E gene ('E-Sarbeco') set (27), the Pasteur Institute RdRp IP2 and IP4 sets ('IP2'

110 and 'IP4', respectively) (28), the Chinese Centre for Disease Control ORF and N gene sets

111 ('China-ORF' and 'China-N', respectively) (29), the Hong Kong University ORF and N gene

112 sets ('HKU-ORF' and 'HKU-N', respectively) (30), and the US-CDC-N1 set (31).

\section{SARS-CoV-2 Synthetic RNA standards}

115 standards comprising six non-overlapping 5,000 base fragments of equal quantities encoding the

116 Wuhan-Hu-1 SARS-CoV-2 genome (Control 2, Genbank ID MN908947.3; Twist Biosciences,

117 supplied at approximately 1 million copies/fragment/ $\mu$ l). To avoid degradation, RNA standards

118 were stored at $-80^{\circ} \mathrm{C}$ and thawed only once, immediately before use, to perform the analytical

119 efficiency, precision, analytical sensitivity and dynamic range analyses described herein.

120 Moreover, to mimic nucleic acid composition of a real biological specimen, all assays employing

121 these standards were supplemented with a consistent, physiologically relevant amount of nucleic

122 acid extracted from pooled remnant SARS-CoV-2-negative nasopharyngeal swabs

123 (Supplementary Figure 1). Briefly, pooled viral transport medium was extracted in 1ml aliquots

124 on the BioMerieux NucliSens ${ }^{\circledR}$ EasyMag ${ }^{\circledR}$, eluted in $60 \mu 1$ and re-pooled. The resulting material

125 contained DNA from on average 2,200 human cells/ $\mu$ (as quantified using human RPP30 DNA

126 copy numbers by ddPCR as described in (32)) and 4,400 human RNAse P copies/ $\mu$ l extract (as 
127 quantified by RT-ddPCR as described in (33)), concentrations that are in line with human DNA

128 and RNA levels recovered on nasopharyngeal swabs $(32,33)$.

129 Reverse transcriptase droplet digital PCR (RT-ddPCR) for SARS-CoV-2 quantification

132 template with target-specific primers and probe $(900 \mathrm{nM}$ and $250 \mathrm{nM}$, respectively, Integrated

133 DNA Technologies; Table 1), One-Step RT-ddPCR Advanced Kit for Probes Supermix, Reverse

134 Transcriptase and DTT (300nM) (all from BioRad), XhoI restriction enzyme (New England

135 Biolabs), background nucleic acid (for reactions employing synthetic RNA template only, see

136 above) and nuclease free water. Droplets were generated using an Automated Droplet Generator

137 (BioRad) and cycled under primer/probe set-specific conditions (see below and Figure 1).

138 Analysis was performed on a QX200 Droplet Reader (BioRad) using QuantaSoft software

139 (BioRad, version 1.7.4).

\section{Thermal cycling temperature optimization}

141 For each primer/probe set, acceptable thermal cycling temperature ranges for reverse

142 transcription (RT) and PCR annealing/extension were determined by modifying the

143 manufacturer-recommended default conditions, which are $42-50^{\circ} \mathrm{C}$ for 1 hour (for reverse

144 transcription); $95^{\circ} \mathrm{C}$ for 10 minutes; 40 cycles of $\left(94^{\circ} \mathrm{C}\right.$ for 30 seconds followed by $50-63^{\circ} \mathrm{C}$ for

1451 minute); $98^{\circ} \mathrm{C}$ for 10 minutes and $4^{\circ} \mathrm{C}$ infinite hold. To determine acceptable temperature

146 ranges for reverse transcription, a thermal gradient from $42-51.5^{\circ} \mathrm{C}$ was performed while fixing

147 the annealing/extension step at $52^{\circ} \mathrm{C}$. Using the optimized reverse transcription temperature, a

148 thermal gradient from $50-63^{\circ} \mathrm{C}$ was then performed to identify acceptable annealing/extension

149 temperature ranges. Temperatures that produced insufficient separation of positive from negative

150 droplets or non-specific amplification were deemed unacceptable, as were those that produced 
151 consecutive $95 \%$ confidence intervals of copy number estimates outside those of the maximal

152 point-estimate.

153 Analytical Efficiency and Precision

154 The analytical efficiency of each primer/probe set to quantify SARS-CoV-2 RNA by RT-

155 ddPCR was determined using synthetic SARS-CoV-2 RNA standards at 1000 and 100 input

156 copies. A minimum of three (maximum four) technical replicates were performed at each

157 concentration. Analytical efficiency was calculated by dividing the measured SARS-CoV-2 copy

158 number by the expected input copy number, and multiplying by 100. Precision was expressed as

159 the coefficient of variation (CV), expressed as a percentage, across technical replicates.

160 Linear Dynamic Range

161 The linear dynamic range (LDR) of each primer/probe set of interest was determined

162 across a serial 1:2 dilution series from 114,286 to 1.2 SARS-CoV-2 RNA copies/reaction. This

163 range of concentrations was chosen as it crosses the entire range of recommended input copies

164 for a ddPCR reaction seeking to quantify the target of interest (34). Reactions were performed in

165 duplicate. The upper and lower limits of quantification of (ULOQ and LLOQ, respectively) were

166 defined as the upper and lower boundaries of the concentration range over which the relationship

167 between measured and input SARS-CoV-2 RNA copies was linear. This was determined by

168 iteratively restricting the range of concentrations included in the linear regression of measured

169 versus input SARS-CoV-2 RNA copies to identify that which maximized the coefficient of

170 determination $\left(\mathrm{R}^{2}\right)$ value and minimized the residuals.

\section{Assay Analytical Sensitivity}

172 Assay analytical sensitivity, defined as the Lower Limit of Detection (LLOD), was

173 determined for primer/probe sets of interest by serially diluting synthetic SARS-CoV-2 RNA 
174 standards to between 47.6 and 0.74 SARS-CoV-2 RNA copies/reaction. Between 6 and 18

175 technical replicates were performed for each dilution and results were analyzed using probit

176 regression. The LLOD, determined through interpolation of the probit curve, was defined as the

177 concentration of input SARS-CoV-2 RNA in a reaction where the probability of detection was

$17895 \%$.

179 SARS-CoV-2 RNA quantification in biological specimens, and relationship to $\mathrm{C}_{\mathrm{t}}$ value

180 Optimized RT-ddPCR assays were applied to a convenience sample of 48 consecutive

181 remnant SARS-CoV-2-positive diagnostic nasopharyngeal swab specimens that were originally

182 submitted to the St. Paul's Hospital Virology Laboratory in Vancouver, Canada for diagnostic

183 testing using the Roche cobas ${ }^{\circledR}$ SARS-CoV-2 assay. For these samples, total nucleic acids were

184 re-extracted from $250 \mu 1$ remnant media using the BioMerieux NucliSens ${ }^{\circledR}$ EasyMag® and

185 eluted in $50 \mu \mathrm{l}$. Eluates were aliquoted and frozen at $-80^{\circ} \mathrm{C}$ prior to single use. SARS-CoV-2 copy

186 numbers were quantified by RT-ddPCR as described above. As our main goal was to

187 characterize the relationship between $\mathrm{C}_{\mathrm{t}}$ values and SARS-CoV-2 RNA levels without

188 confounding by extraction platform, quantity of input material or SARS-CoV-2 genomic target,

189 we re-tested these extracts using a commercial real-time RT-PCR SARS-CoV-2 diagnostic assay

190 that uses the E-Sarbeco primer/probe set (27): the LightMix ${ }^{\circledR}$ 2019-nCoV real-time RT-PCR

191 assay E-gene target (Tib-Molbiol), implemented on LightCycler 480 (Roche Diagnostics).

192 Finally, to be responsive to a recent recommendation that SARS-CoV-2 viral loads be reported

193 in terms of SARS-CoV-2 RNA copies per human cell equivalents (9), we measured human

194 cells/ $\mu 1$ extract by ddPCR as previously described (32) and additionally reported results as

195 SARS-CoV-2 RNA copies/1,000 human cells.

\section{Statistical Analysis}


197 Statistical analysis was performed using GraphPad Prism (Version 8) or Microsoft Excel

198 (Version 14.7.2).

199 Ethical Approval

200 This study was approved by the Providence Health Care/University of British Columbia

201 and Simon Fraser University Research Ethics Boards under protocol H20-01055. 


\section{Results}

\section{Thermal cycling optimization for SARS-CoV-2 quantification by RT-ddPCR}

205 Eight primer/probe sets originally developed for SARS-CoV-2 diagnostic testing by real

206 time RT-PCR were evaluated for use in RT-ddPCR (Table 1). As these primer/probe sets vary in

207 sequence, amplicon length and SARS-CoV-2 genomic target, we first determined the acceptable

208 temperature ranges for reverse transcription (RT) and PCR annealing/extension. Most

209 primer/probe sets were tolerant to a wide temperature range, and background signal was

210 essentially zero at all temperatures tested (Figure 1). The E-Sarbeco primer/probe set for

211 example produced consistent amplitude profiles, copy number estimates and essentially zero

212 background at annealing/extension temperatures ranging from $50-63^{\circ} \mathrm{C}$ (Figure $1 \mathrm{~A}$ and data not

213 shown). The HKU-ORF primer/probe performed acceptably over a $50-60.5^{\circ} \mathrm{C}$

214 annealing/extension range, but positive and negative droplet separation was insufficient at higher

215 temperatures (Figure 1B). Acceptable temperature ranges for each primer/probe set are shown in

216 Figure 1C. All subsequent experiments were performed at RT $42.7^{\circ} \mathrm{C}$ and annealing/extension

$21750.9^{\circ} \mathrm{C}$ except those for HKU-ORF and US-CDC-N1, which were performed at RT $45.7^{\circ} \mathrm{C}$ and

218 annealing/extension $55.1^{\circ} \mathrm{C}$ as informed by initial qualitative assessments.

219 Analytical Efficiency and Precision of SARS-CoV-2 quantification by RT-ddPCR

220 We next evaluated the analytical efficiency of SARS-CoV-2 RNA quantification for each

221 primer/probe set, calculated as the percentage of input viral RNA copies detected by the assay.

222 We also evaluated precision, calculated as the dispersion of measured copies around the mean

223 (coefficient of variation, CV). Analytical efficiency and precision were evaluated at 1000 and

224100 SARS-CoV-2 RNA target input copies. At 1000 input copies, primer/probe set analytical

225 efficiency ranged from 83\% (E-Sarbeco) to 15\% (US-CDC-N1) (Figure 2A). At 100 copies, the 
226 analytical efficiency hierarchy was identical, with values ranging from $74 \%$ (E Sarbeco) to $12 \%$

227 (US-CDC-N1). Of all primer/probe sets evaluated, the E-Sarbeco, IP2 and IP4 sets had the

228 highest analytical efficiencies by a substantial margin. At 1000 and 100 target copies, E-Sarbeco

229 analytical efficiency was 83\% (95\% Total Poisson Confidence Interval [CI]: 79- 87\%) and 74\%

230 (95\% CI: 63- 84\%), respectively; IP2, analytical efficiency was 70\% (95\% CI: 67- 73\%) and

231 55\% (95\% CI: 46- 64\%), respectively; and IP4 analytical efficiency was 69\% (95\% CI: 66- 72\%)

232 and 59\% (95\% CI: 50-69\%), respectively. In contrast, analytical efficiency of the China-ORF

233 primer/probe set was only $46 \%$ and $39 \%$ at 1000 and 100 input copies, respectively, and the

234 analytical efficiencies of the remaining sets were less than $30 \%$ regardless of input copy number.

235 Furthermore, while measurement precision generally decreased at the lower template

236 concentration (35), the E-Sarbeco, IP2 and IP4 primer/probe sets were nevertheless among the

237 most precise, with coefficients of variation (CV) of less than 5\% at 1,000 input copies and less

238 than $15 \%$ at 100 input copies (Figure 2B). Combined analytical efficiency and precision data

239 confirmed E-Sarbeco, IP2 and IP4 as the best-performing primer/probe sets in RT-ddPCR

240 (Figures 2C and 2D), so these were moved forward for further characterization.

241 Reduced analytical efficiency when IP2 and IP4 are duplexed in RT-ddPCR

242 As IP2 and IP4 were originally designed for duplexing in real-time RT-PCR (28), we

243 evaluated them in duplex for RT-ddPCR. Duplexing however decreased analytical efficiency,

244 from $70 \%$ to $52 \%$ (at 1000 input copies) and 55\% to $37 \%$ (at 100 input copies) for IP2, and from

$24569 \%$ to $49 \%$ (at 1000 input copies) and 59\% to 38\% (at 100 input copies) for IP4 (Supplemental

246 Figure 2A). Duplexing also decreased precision (Supplemental Figure 2B). For IP2, CV

247 increased from 5\% to $11 \%$ when duplexing at 1000 input copies, and from $15 \%$ to $25 \%$ when

248 duplexing at 100 input copies. For IP4, CV increased from 4\% to 7\% (1000 input copies) and 
249 from $14 \%$ to $21 \%$ (100 input copies) with duplexing. Duplexing of these reactions is therefore

250 not recommended in RT-ddPCR, and all IP2 and IP4 assays were performed as single reactions.

251 Linear Dynamic Range and Limits of Quantification of SARS-CoV-2 RNA by RT-ddPCR

252 Droplet digital PCR can achieve absolute target copy number quantification without a

253 standard curve. To investigate the linear dynamic range (LDR) of quantification of the E-

254 Sarbeco, IP2 and IP4 assays, we set up 18 two-fold serial dilutions of synthetic SARS-CoV-2

255 RNA beginning at 114,286 copies/reaction (this copy number is obtained when 120,000 copies

256 are added to a $21 \mu 1$ reaction, of which $20 \mu 1$ is used for droplet generation) and ending with 2.32

257 copies/reaction. This input copy number range crosses nearly the entire manufacturer-

258 recommended template input range for ddPCR reactions seeking to quantify the target of interest,

259 which is $1-100,000$ copies/reaction (36).

261 concentrations included in the linear regression of measured versus input SARS-CoV-2 RNA

262 copies to identify the range that maximized the $\mathrm{R}^{2}$ value and minimized the residuals. For E-

263 Sarbeco, the regression spanning 18.6-114,286 input SARS-CoV-2 RNA copies per reaction, an

264 approximately 6,100-fold concentration range, yielded an $\mathrm{R}^{2}$ value of 0.9995 (Figure $3 \mathrm{~A}$, left).

265 Restricting the linear regression to this range also minimized the residuals of all included data

266 points to $\pm 0.065 \log _{10}$ copies/reaction (Figure 3A, right). The IP2 assay, while less efficient than

267 E-Sarbeco, had the same estimated LDR of 18.6-114,286 input copies/reaction (Figure 3B, left).

268 This produced an $\mathrm{R}^{2}$ value of 0.9995 and residuals within $\pm 0.065 \log _{10}$ copies/reaction across the

269 LDR (Figure 3B, right). The LDR of IP4 was estimated as 37.2- 114,286 input copies/reaction,

270 an approximately 3,000-fold range, which yielded an $\mathrm{R}^{2}=0.9975$ and produced residuals within

$271 \pm 0.11 \log _{10}$ copies/reaction across this range (Figure 3C). For all three assays, 114,286 input 
272 copies/reaction should be considered a conservative estimate of the upper limit of quantification,

273 as saturation of the RT-ddPCR reaction or loss of linearity was still not achieved at this

274 concentration.

\section{Lower Limit of Detection of SARS-CoV-2 RNA by RT-ddPCR}

277 RT-ddPCR assays (Figure 4). Probit regression analysis applied to serial dilutions of synthetic

278 SARS-CoV-2 RNA standards revealed the E-Sarbeco RT-ddPCR assay to be the most

279 analytically sensitive of the three, which is consistent with it also having the highest analytical

280 efficiency. Specifically, the estimated LLOD of the E-Sarbeco assay was 4.4 (95\% Confidence

281 Interval [CI]: 2.4-5.7) SARS-CoV-2 RNA copies/reaction (Figure 4A). The estimated LLOD of

282 the IP2 assay was 7.8 (95\% CI: 4.4-10.3) SARS-CoV-2 RNA copies/reaction (Figure 4B), while

283 that of IP4 was 12.6 (95\% CI: 6.9-16.5) SARS-CoV-2 RNA copies per reaction (Figure 4C).

284 SARS-CoV-2 viral loads in biological samples using the E-Sarbeco, IP2 and IP4 primer/probe sets (note that samples with original diagnostic test $\mathrm{C}_{\mathrm{t}}$ values $<19$ required RNA extracts to be diluted up to 1:200 prior to quantification to ensure that input copies measurements fell within each assay's LDR). The results revealed that

289 SARS-CoV-2 RNA in these biological samples varied over a $6.2 \log _{10}$ range (Figure 5A).

290 Average copy numbers measured using the E-Sarbeco assay (which targets the E gene) were

291 higher than those using the IP2 and IP4 assays (which target ORF1a and ORF1b, respectively)

292 (Figure 5A). This is consistent with assay analytical efficiency (Figure 2) and in vivo coronavirus

293 RNA expression patterns, where transcripts covering the 3' end of the genome are more

294 abundant than those covering the 5' end (37-40). Specifically, the median E-gene copy number 
was 5.1 (IQR 3.9- 5.7) $\log _{10}$ copies/ $\mu$ l extract compared to a median of 4.9 (IQR 3.9- 5.5)

$296 \log _{10}$ copies $/ \mu 1$ extract for the IP2 target, and a median of 4.9 (IQR 3.9- 5.6) $\log _{10} \operatorname{copies} / \mu 1$

297 extract for the IP4 target. SARS-CoV-2 E-gene, IP2 and IP4 copy numbers in biological samples

298 correlated strongly with one another (Spearman's $\rho>0.99 ; p<0.0001$ for all pairwise analyses;

299 Figure 5BCD). Consistent with comparable ORF1a and ORF1b RNA transcript levels in vivo

$300(37,38,40)$, IP2 and IP4 copy numbers were also highly concordant (Lin's concordance

301 correlation coefficient, $\rho c=0.9996$ [95\% CI: 0.9993- 0.9998]) (Figure 5D). Based on a recent

302 recommendation (9), we also report our results in terms of SARS-CoV-2 RNA copies per human

303 cell equivalents: results for E-Sarbeco spanned an 7-fold range from 1.05 to $7.3 \log _{10}$ SARS-

304 CoV-2 RNA copies/1,000 human cells, with IP2 and IP4 $\log _{10}$ copy numbers lower, as expected

305 (Supplemental Figure 3A). The Spearman's correlation between absolute and human cell-

306 normalized viral loads was strong $(\rho=0.9717$; $p<0.0001$; Supplementary Figure $3 B)$, which is

307 consistent with the assumption that the amount of biological material collected by

308 nasopharyngeal swabs is relatively consistent.

309 Inferring SARS-CoV-2 viral loads from diagnostic $\mathbf{C}_{t}$ values

310 Finally, we characterized the relationship between $\mathrm{C}_{\mathrm{t}}$ values produced by a commercial

311 COVID-19 diagnostic platform and SARS-CoV-2 RNA copy numbers. We selected the

312 LightMix ${ }^{\circledR}$ 2019-nCoV real-time RT-PCR assay, E-gene target (Tib-Molbiol), implemented on a

313 LightCycler 480 (Roche Diagnostics) because commercial diagnostic reagents comprising the E-

314 Sarbeco primer/probe set exist for this platform (27) and because it takes purified nucleic acids

315 as input, thereby allowing direct comparison of results from the same starting material (real-time

316 RT-PCR platforms that take biological material as input are suboptimal for such a comparison

317 because the onboard extraction introduces an additional variable). As the $C_{t}$ values reported for 
318 the LightMix ${ }^{\circledR}$ assay are based on a $9 \mu$ l extract input volume, our primary analysis reported RT-

319 ddPCR results in terms of SARS-CoV-2 copies equivalent (i.e. SARS-CoV-2 copies in $9 \mu 1$ of

320 extract), to allow direct conversion of $\mathrm{C}_{\mathrm{t}}$ values to absolute viral copy numbers.

321 Sample $C_{t}$ values ranged from 11.34-31.18 (median 18.69 [IQR 16.73- 22.69]) using the

322 LightMix ${ }^{\circledR}$ assay. The relationship between $C_{t}$ value and SARS-CoV-2 RNA copy numbers was

323 log-linear, with an $\mathrm{R}^{2}=0.9990$ (Figure 6). Despite this strong relationship, inspection of the

324 residuals nevertheless suggested modest departures from log-linearity at the extremes of the

325 linear range (Supplementary Figure 4). The relationship between $C_{t}$ value and absolute SARS-

326 CoV-2 E-gene copies can thus be given by $\log _{10}$ SARS-CoV-2 E gene copies equivalent $=$

$327-0.3038 \mathrm{C}_{\mathrm{t}}+11.7$ (Figure 6). That is, a $\mathrm{C}_{\mathrm{t}}$ value of 20 corresponds to 453,942 (i.e. $\left.5.66 \log _{10}\right)$

328 SARS-CoV-2 RNA copies, while a $\mathrm{C}_{\mathrm{t}}$ value of 30 corresponds to 416 (i.e. $2.62 \log _{10}$ ) viral

329 copies. This equation also predicts that the $\mathrm{C}_{\mathrm{t}}$ values corresponding to the LLOQ and LLOD of

330 the E-Sarbeco RT-ddPCR assays are 34.8 and 36.84, respectively. When measured SARS-CoV-2

331 RNA copy numbers are expressed as human cell-normalized viral loads, the relationship with $\mathrm{Ct}$

332 value is given by $\log _{10} \mathrm{SARS}-\mathrm{CoV}-2 \mathrm{E}$ gene copies $/ 1,000$ human cells $=-0.3041 \mathrm{C}_{\mathrm{t}}+10.8$

333 (Supplemental Figure 5). An extract that yielded a $C_{t}$ value of 20 therefore is estimated to have

334 contained 48,978 (i.e. $4.69 \log _{10}$ ) SARS-CoV-2 RNA copies/1,000 human cells, while one with

$335 \mathrm{C}_{\mathrm{t}}$ value of 30 is estimated to have contained 45 (i.e. $1.66 \log _{10}$ ) copies/1,000 human cells

337 Discussion

338 While real-time and droplet digital RT-PCR platforms both employ target-specific

339 primers coupled with fluorescence-based amplicon detection, there are key differences in

340 reaction chemistry (e.g. RT-ddPCR reagents must be compatible with water-in-oil droplet 
341 partitioning) and probe chemistry (e.g. while real-time RT-PCR uses fluorescent quenchers,

342 ddPCR typically uses dark quenchers). As a result, assays developed for one platform may not

343 always translate seamlessly to the other. For example, ddPCR probes should ideally not have a

344 Guanine at their 5' end because this quenches the fluorescence signal even following hydrolysis

345 (36) but the HKU-N probe has a G at its 5' end (Table 1).

346 It is perhaps therefore not surprising that the overall performance of the eight

347 primer/probe sets in RT-ddPCR did not exactly mirror that in real-time RT-PCR $(41,42)$.

348 Nevertheless, E-Sarbeco, IP2 and IP4, which represented the most efficient and precise

349 primer/probe sets for SARS-CoV-2 RNA quantification by RT-ddPCR are also among the most

350 efficient in real-time RT-PCR $(41,42)$. Our results also confirm previous reports of the E-

351 Sarbeco primer/probe set performing well in RT-ddPCR $(10,22)$. Other primer/probe sets

352 however, notably US CDC-N1, HKU-ORF and China-ORF, did not perform as well in our RT-

353 ddPCR assay compared to a previous report (10). One key difference is that, while we used

354 sequence-specific reverse transcription (with the reverse primer) in a one-step RT-ddPCR

355 reaction, the previous study featured an independent reverse transcription reaction primed with

356 random hexamers and oligo dT, which can yield higher efficiency than sequence-specific

357 priming $(35,43-45)$, to generate cDNA for input into a ddPCR reaction. To our knowledge, ours

358 is the first study to evaluate IP2 or IP4 primer/probe sets in RT-ddPCR.

359 The analytical sensitivities of the RT-ddPCR assays reported here are nevertheless

360 comparable to existing estimates. The limit of detection of the BioRad SARS-CoV-2 ddPCR Kit

361 (20) is, for example, estimated at 150 copies/mL, which is comparable to our E-Sarbeco RT-

362 ddPCR assay (estimated at 75.8 copies/mL assuming 100\% extraction efficiency). Similarly, the

363 LLODs of the TargetingOne (Beijing, China) COVID-19 digital PCR detection kit (23) and a 
364 multiplex assay that included the E-Sarbeco primer/probe set (22) were reported at 10 copies/test

365 and 5 copies/reaction, respectively, both comparable to the LLOD determined here. While a

366 number of studies have reported that RT-ddPCR can detect SARS-CoV-2 RNA in low viral load

367 clinical samples with higher sensitivity than real-time RT-PCR $(11,21,23-25)$, our study was

368 not designed to evaluate this. Our estimated LLOD of 4.4 copies/reaction by RT-ddPCR using

369 the E-Sarbeco primer/probe set (Figure 4) is in fact comparable to the LLOD reported for many

370 real-time RT-PCR-based COVID-19 diagnostic assays (46).

371 The ability to quantify SARS-CoV-2 viral loads in biological samples can advance our

372 understanding of COVID-19 biology, and RT-ddPCR offers an attractive platform (7, 8). Our

373 observation that, in a small convenience sample, both absolute and human cell-normalized (9)

374 SARS-CoV-2 loads spanned a more than $6 \log _{10}$ range confirms an enormous viral load range in

375 vivo (47) and suggests that some of the high viral load samples measured here were from

376 individuals with early and progressive infection $(23,48-50)$ or who were experiencing severe

377 disease $(7,8)$, though clinical information was unknown. Furthermore, our equation relating $\mathrm{C}_{\mathrm{t}}$

378 values derived from a commercial diagnostic assay and SARS-CoV-2 RNA copy number means

379 that existing diagnostic test results can be converted to viral loads without re-testing samples.

380 While calibration of viral load measurements against all real-time RT-PCR platforms is beyond

381 our scope, this is achievable and in some cases data may already be available (23).

382 Some limitations merit mention. We only tested eight commonly-used SARS-CoV-2-

383 specific primer/probe sets, and others may exist that adapt well to RT-ddPCR. Our assay

384 performance estimates should be considered approximate, as the manufacturer-reported

385 concentration of the synthetic SARS-CoV-2 RNA standards used in our study may vary by up to

$38620 \%$ error (Twist Bioscience, personal communication). Moreover, we solely evaluated a one- 
387 step RT-ddPCR protocol, and therefore assay performance estimates will likely differ from

388 protocols that feature independent cDNA generation followed by ddPCR. We could not precisely

389 define the upper boundary of the linear dynamic range of the E-Sarbeco, IP2 and IP4 RT-ddPCR

390 assays as linearity was maintained at the maximum input of 114,286 target copies/reaction,

391 which already exceeds the manufacturer's estimated upper range of quantification in a ddPCR

392 reaction (36). Our convenience panel of 48 SARS-CoV-2-positive diagnostic specimens also

393 likely did not capture the full range of biological variation in viral loads, though data from larger

394 cohorts (47) suggests that it was reasonably comprehensive. We also acknowledge that there is

395 measurement uncertainty with real-time RT-PCR $C_{t}$ values that may subtly affect the linear

396 relationship between $\mathrm{C}_{\mathrm{t}}$ value and RT-ddPCR-derived SARS-CoV-2 viral load described here.

397 Finally, our estimates of assay performance may not completely reflect those of the entire

398 diagnostic process, as the nucleic acid extraction step introduces additional inefficiencies.

399 In conclusion, primer/probe sets used in real-time RT-PCR-based COVID-19 diagnostic

400 tests can be migrated to RT-ddPCR to achieve SARS-CoV-2 RNA quantification with varying

401 analytical efficiency, precision and sensitivity. Of the primer/probe sets tested, the E-Sarbeco,

402 IP2 and IP4 sets performed best, where LLOQ and LLOD estimates for the E-Sarbeco assay

403 (18.6 and 4.4 copies/reaction, respectively) indicated that RT-ddPCR and real-time RT-PCR

404 have comparable sensitivity. Mathematical inference of SARS-CoV-2 copy numbers from

405 COVID-19 diagnostic test $\mathrm{C}_{\mathrm{t}}$ values, made possible via the type of calibration performed in the

406 present study, will allow the wealth of existing diagnostic test data to be harnessed to answer

407 foundational questions in SARS-CoV-2 biology.

408

409 Acknowledgements 
410 This work was supported by a COVID-19 rapid response grant from GenomeBC

411 (COVID-115; to ZLB and CFL) and CIHR project grant (PJT-159625; to ZLB). NNK is

412 supported by a Vanier Canada Graduate Scholarship. ZLB holds a Scholar Award from the

413 Michael Smith Foundation for Health Research.

414

415 
1. Mellors JW, Rinaldo CR, Jr., Gupta P, White RM, Todd JA, Kingsley LA. 1996. Prognosis in HIV-1 infection predicted by the quantity of virus in plasma. Science 272:1167-70.

2. Mellors JW. 1998. Viral-load tests provide valuable answers. Sci Am 279:90-3.

3. Riddler SA, Mellors JW. 1997. HIV-1 viral dynamics and viral load measurement: implications for therapy. AIDS Clin Rev:47-65.

4. Mellors JW, Muñoz A, Giorgi JV, Margolick JB, Tassoni CJ, Gupta P, Kingsley LA, Todd JA, Saah AJ, Detels R, Phair JP, Rinaldo CR, Jr. 1997. Plasma viral load and CD4+ lymphocytes as prognostic markers of HIV-1 infection. Ann Intern Med 126:946-54.

5. Durante-Mangoni E, Zampino R, Portella G, Adinolfi LE, Utili R, Ruggiero G. 2009. Correlates and prognostic value of the first-phase hepatitis $\mathrm{C}$ virus RNA kinetics during treatment. Clin Infect Dis 49:498-506.

6. Chen G, Lin W, Shen F, Iloeje UH, London WT, Evans AA. 2006. Past HBV viral load as predictor of mortality and morbidity from HCC and chronic liver disease in a prospective study. Am J Gastroenterol 101:1797-803.

7. Veyer D, Kernéis S, Poulet G, Wack M, Robillard N, Taly V, L'Honneur AS, Rozenberg F, Laurent-Puig P, Bélec L, Hadjadj J, Terrier B, Péré H. 2020. Highly sensitive quantification of plasma SARS-CoV-2 RNA shelds light on its potential clinical value. Clin Infect Dis doi:10.1093/cid/ciaa1196.

8. Bermejo-Martin JF, González-Rivera M, Almansa R, Micheloud D, Tedim AP, Domínguez-Gil M, Resino S, Martín-Fernández M, Murua PR, Pérez-García F, Tamayo L, Lopez-Izquierdo R, Bustamante E, Aldecoa C, Gómez JM, Rico-Feijoo J, Orduña A, Méndez R, Fernández Natal I, Megías G, González-Estecha M, Carriedo D, Doncel C, Jorge N, Ortega A, de la Fuente A, del Campo F, Fernández-Ratero JA, Trapiello W, González-Jiménez P, Ruiz G, Kelvin AA, Ostadgavahi AT, Oneizat R, María Ruiz L, Miguéns I, Gargallo E, Muñoz I, Pelegrin S, Martín S, García Olivares P, Cedeño JA, Albi TR, Puertas C, Ángel Berezo J, Renedo G, Herrán R, Bustamante-Munguira J, Enríquez P, Cicuendez R, et al. 2020. Viral RNA load in plasma is associated with critical illness and a dysregulated host response in COVID-19. medRxiv doi:10.1101/2020.08.25.20154252:2020.08.25.20154252.

9. Han MS, Byun JH, Cho Y, Rim JH. 2020. RT-PCR for SARS-CoV-2: quantitative versus qualitative. Lancet Infect Dis doi:10.1016/s1473-3099(20)30424-2.

10. Liu X, Feng J, Zhang Q, Guo D, Zhang L, Suo T, Hu W, Guo M, Wang X, Huang Z, Xiong Y, Chen G, Chen Y, Lan K. 2020. Analytical comparisons of SARS-COV-2 detection by qRT-PCR and ddPCR with multiple primer/probe sets. Emerg Microbes Infect 9:1175-1179.

11. Suo T, Liu X, Feng J, Guo M, Hu W, Guo D, Ullah H, Yang Y, Zhang Q, Wang X, Sajid M, Huang Z, Deng L, Chen T, Liu F, Xu K, Liu Y, Zhang Q, Liu Y, Xiong Y, Chen G, Lan K, Chen Y. 2020. ddPCR: a more accurate tool for SARS-CoV-2 detection in low viral load specimens. Emerg Microbes Infect 9:1259-1268.

12. BioRad-Laboratories-Inc. 2020. Wet-Lab Validated ddPCR Assays for Mutation Detection and Copy Number Determination. https://www.bio- 
13. BioRad-Laboratories-Inc. 2019. Direct Quantification of Genome Editing Efficiency 
507 26. World-Health-Organization. 2020. Summary of Available SARS-CoV-2 RT-PCR

$508 \quad$ protocols.

509 27. Corman VM, Landt O, Kaiser M, Molenkamp R, Meijer A, Chu DK, Bleicker T, Brünink

510

511

512

513

514

515

516

517

518

519

520

521

522

523

524

525

526

527

528

529

530

531

532

533

534

535

536

537

538

539

540

541

542

543

544

545

546

547

548

549

550 S, Schneider J, Schmidt ML, Mulders DG, Haagmans BL, van der Veer B, van den Brink S, Wijsman L, Goderski G, Romette JL, Ellis J, Zambon M, Peiris M, Goossens H, Reusken C, Koopmans MP, Drosten C. 2020. Detection of 2019 novel coronavirus (2019-nCoV) by real-time RT-PCR. Euro Surveill 25.

28. Institut-Pasteur-Paris. 2020. Protocol: Real-time RT-PCR assays for the detection of SARS-CoV-2.

29. Chinese-National-Institute-for-Viral-Disease-Control-and-Prevention. 2020. Specific primers and probes for detection of 2019 novel coronavirus.

30. School-of-Public-Health-LKS-Faculty-of-Medicine-University-of-Hong-Kong. 2020. Detection of 2019 novel coronavirus (2019-nCoV) in suspected human cases by RTPCR.

31. Centers-for-Disease-Control-and-Prevention. 2020. CDC 2019-Novel Coronavirus (2019-nCoV) Real-Time RT-PCR Diagnostic Panel.

32. Kinloch NN, Ritchie G, Brumme CJ, Dong W, Dong W, Lawson T, Jones RB, Montaner JSG, Leung V, Romney MG, Stefanovic A, Matic N, Lowe CF, Brumme ZL. 2020. Suboptimal Biological Sampling as a Probable Cause of False-Negative COVID-19 Diagnostic Test Results. The Journal of Infectious Diseases doi:10.1093/infdis/jiaa370.

33. Kinloch NN, Shahid A, Ritchie G, Dong W, Lawson T, Montaner JSG, Romney MG, Stefanovic A, Matic N, Brumme CJ, Lowe CF, Brumme ZL, Leung V. 2020. Evaluation of Nasopharyngeal Swab Collection Techniques for Nucleic Acid Recovery and Participant Experience: Recommendations for COVID-19 Diagnostics. Open Forum Infect Dis 7:ofaa488.

34. Bio-Rad-Laboratories-Inc. 2019. Droplet Digital PCR Applications Guide.

35. Schwaber J, Andersen S, Nielsen L. 2019. Shedding light: The importance of reverse transcription efficiency standards in data interpretation. Biomol Detect Quantif 17:100077.

36. BioRad-Laboratories-Inc. 2019. Droplet Digital PCR Applications Guide. https://www.bio-rad.com/webroot/web/pdf/lsr/literature/Bulletin_6407.pdf. Accessed

37. Kim D, Lee JY, Yang JS, Kim JW, Kim VN, Chang H. 2020. The Architecture of SARSCoV-2 Transcriptome. Cell 181:914-921.e10.

38. Irigoyen N, Firth AE, Jones JD, Chung BY, Siddell SG, Brierley I. 2016. HighResolution Analysis of Coronavirus Gene Expression by RNA Sequencing and Ribosome Profiling. PLoS Pathog 12:e1005473.

39. Dimmock NJ, Easton AJ, Leppard KN. 2007. Introduction to Modern Virology, 6 ed. Blackwell Publishing.

40. Davidson AD, Williamson MK, Lewis S, Shoemark D, Carroll MW, Heesom KJ, Zambon M, Ellis J, Lewis PA, Hiscox JA, Matthews DA. 2020. Characterisation of the transcriptome and proteome of SARS-CoV-2 reveals a cell passage induced in-frame deletion of the furin-like cleavage site from the spike glycoprotein. Genome Med $551 \quad 12: 68$. 
552 41. Vogels CBF, Brito AF, Wyllie AL, Fauver JR, Ott IM, Kalinich CC, Petrone ME,

553

554

555

556

557

558

559

560

561

562

563

564

565

566

567

568

569

570

571

572

573

574

575

576

577

578

579

580

581

582

583

584

585

586
Casanovas-Massana A, Catherine Muenker M, Moore AJ, Klein J, Lu P, Lu-Culligan A, Jiang X, Kim DJ, Kudo E, Mao T, Moriyama M, Oh JE, Park A, Silva J, Song E, Takahashi T, Taura M, Tokuyama M, Venkataraman A, Weizman OE, Wong P, Yang Y, Cheemarla NR, White EB, Lapidus S, Earnest R, Geng B, Vijayakumar P, Odio C, Fournier J, Bermejo S, Farhadian S, Dela Cruz CS, Iwasaki A, Ko AI, Landry ML, Foxman EF, Grubaugh ND. 2020. Analytical sensitivity and efficiency comparisons of SARS-CoV-2 RT-qPCR primer-probe sets. Nat Microbiol 5:1299-1305.

42. Etievant S, Bal A, Escuret V, Brengel-Pesce K, Bouscambert M, Cheynet V, Generenaz L, Oriol G, Destras G, Billaud G, Josset L, Frobert E, Morfin F, Gaymard A. 2020. Performance Assessment of SARS-CoV-2 PCR Assays Developed by WHO Referral Laboratories. J Clin Med 9.

43. Bustin SA, Benes V, Nolan T, Pfaffl MW. 2005. Quantitative real-time RT-PCR--a perspective. J Mol Endocrinol 34:597-601.

44. Sanders R, Mason DJ, Foy CA, Huggett JF. 2013. Evaluation of digital PCR for absolute RNA quantification. PLoS One 8:e75296.

45. Levesque-Sergerie JP, Duquette M, Thibault C, Delbecchi L, Bissonnette N. 2007. Detection limits of several commercial reverse transcriptase enzymes: impact on the low- and high-abundance transcript levels assessed by quantitative RT-PCR. BMC Mol Biol 8:93.

46. Lai CC, Wang CY, Ko WC, Hsueh PR. 2020. In vitro diagnostics of coronavirus disease 2019: Technologies and application. J Microbiol Immunol Infect doi:10.1016/j.jmii.2020.05.016.

47. Jacot D, Greub G, Jaton K, Opota O. 2020. Viral load of SARS-CoV-2 across patients and compared to other respiratory viruses. Microbes Infect doi:10.1016/j.micinf.2020.08.004.

48. Pan Y, Zhang D, Yang P, Poon LLM, Wang Q. 2020. Viral load of SARS-CoV-2 in clinical samples. Lancet Infect Dis 20:411-412.

49. Zou L, Ruan F, Huang M, Liang L, Huang H, Hong Z, Yu J, Kang M, Song Y, Xia J, Guo Q, Song T, He J, Yen HL, Peiris M, Wu J. 2020. SARS-CoV-2 Viral Load in Upper Respiratory Specimens of Infected Patients. N Engl J Med 382:1177-1179.

50. Huang Y, Chen S, Yang Z, Guan W, Liu D, Lin Z, Zhang Y, Xu Z, Liu X, Li Y. 2020. SARSCoV-2 Viral Load in Clinical Samples from Critically Ill Patients. Am J Respir Crit Care Med 201:1435-1438. 


\begin{tabular}{|c|c|c|c|c|c|}
\hline Source & Name & $\begin{array}{c}\text { Gene } \\
\text { Target } \\
\end{array}$ & $\begin{array}{c}\text { Primer/ } \\
\text { Probe }\end{array}$ & $\begin{array}{c}\text { Sequence }^{\phi} \\
\left(5^{\prime}->3^{\prime}\right)\end{array}$ & Coordinates $^{\psi}$ \\
\hline \multirow{3}{*}{$\begin{array}{c}\text { Charité- } \\
\text { Berlin }\end{array}$} & \multirow{3}{*}{ E-Sarbeco } & \multirow{3}{*}{$\mathrm{E}$} & Fwd Primer & ACAGGTACGTTAATAGTTAATAGCGT & $26,269-26,294$ \\
\hline & & & Rev Primer & ATATTGCAGCAGTACGCACACA & $26,381-26,360$ \\
\hline & & & Probe & FAM-ACACTAGCC/ZEN/ATCCTTACTGCGCTTCG-3IABkFQ & $26,332-26,357$ \\
\hline \multirow{6}{*}{$\begin{array}{l}\text { Pasteur } \\
\text { Institute }\end{array}$} & \multirow{3}{*}{ IP2 } & \multirow{3}{*}{ ORF1a } & Fwd Primer & ATGAGCTTAGTCCTGTTG & $12,690-12,707$ \\
\hline & & & Rev Primer & CTCCCTTTGTTGTGTTGT & $12,797-12,780$ \\
\hline & & & Probe & HEX-AGATGTCTT/ZEN/GTGCTGCCGGTA-3IABkFQ & $12,717-12,737$ \\
\hline & \multirow{3}{*}{ IP4 } & \multirow{3}{*}{ ORF1b } & Fwd Primer & GGTAACTGGTATGATTTCG & $14,080-14,098$ \\
\hline & & & Rev Primer & CTGGTCAAGGTTAATATAGG & $14,105-14,123$ \\
\hline & & & Probe & FAM-TCATACAAA/ZEN/CCACGCCAGG-3IABkFQ & $14,186-14,167$ \\
\hline \multirow{6}{*}{ China CDC } & \multirow{3}{*}{ China-ORF } & \multirow{3}{*}{ ORF1a } & Fwd Primer & CCCTGTGGGTTTTACACTTAA & $13,342-13,362$ \\
\hline & & & Rev Primer & ACGATTGTGCATCAGCTGA & $13,460-13,442$ \\
\hline & & & Probe & FAM-CCGTCTGCG/ZEN/GTATGTGGAAAGGTTATGG-3IABkFQ & $13,377-13,404$ \\
\hline & \multirow{3}{*}{ China-N } & \multirow{3}{*}{$\mathrm{N}$} & Fwd Primer & GGGGAACTTCTCCTGCTAGAAT & $28,881-28,902$ \\
\hline & & & Rev Primer & CAGACATTTTGCTCTCAAGCTG & $28,979-28,958$ \\
\hline & & & Probe & FAM-TTGCTGCTG/ZEN/CTTGACAGATT-3IABkFQ & $28,934-28,953$ \\
\hline \multirow{6}{*}{$\begin{array}{l}\text { Hong Kong } \\
\text { University }\end{array}$} & \multirow{3}{*}{ HKU-ORF } & \multirow{3}{*}{ ORF1b } & Fwd Primer & TGGGGYTTTACRGGTAACCT & $18,778-18,797$ \\
\hline & & & Rev Primer & AACRCGCTTAACAAAGCACTC & $18,849-18,872$ \\
\hline & & & Probe & FAM-TAGTTGTGA/ZEN/TGCWATCATGACTAG-3IABkFQ & $18,909-18,889$ \\
\hline & \multirow{3}{*}{ HKU-N } & \multirow{3}{*}{$\mathrm{N}$} & Fwd Primer & TAATCAGACAAGGAACTGATTA & $29,145-29,166$ \\
\hline & & & Rev Primer & CGAAGGTGTGACTTCCATG & $29,179-29,198$ \\
\hline & & & Probe & FAM-GCAAATTGT/ZEN/GCAATTTGCGG-3IABkFQ & $29,254-29,236$ \\
\hline \multirow{3}{*}{ US CDC } & \multirow{3}{*}{ US-CDC-N1 } & \multirow{3}{*}{$\mathrm{N}$} & Fwd Primer & GACCCCAAAATCAGCGAAAT & $28,287-28,306$ \\
\hline & & & Rev Primer & TCTGGTTACTGCCAGTTGAATCTG & $28,358-28,335$ \\
\hline & & & Probe & FAM-ACCCCGCAT/ZEN/TACGTTTGGTGGACC-3IABkFQ & $28,309-28,332$ \\
\hline
\end{tabular}


Figure 1: Thermal cycling optimization (A). RT-ddPCR plots for annealing/extension under a $50-63^{\circ} \mathrm{C}$ thermal gradient for the E-Sarbeco primer/probe set. A representative RT-ddPCR plot for a no template control (NTC) which only included non-target DNA/RNA (see methods) at the temperature used in subsequent experiments, is also shown. Positive droplets (blue) are above the threshold (pink line); negative droplets (grey) are below the line. Colored boxes below each well indicate if results met standards for inclusion (green) or not (red) (see methods). (B). Same as panel A, but for HKU-ORF primer/probe set. (C). Acceptable RT and annealing/extension temperature ranges for each primer/probe set.

Figure 2: Analytical efficiency and precision of primer/probe sets. (A) Analytical efficiency of each primer/probe set, calculated as the measured divided by the input SARS-CoV-2 RNA copies multiplied by $100 \%$, is shown for reactions containing 1,000 and 100 input copies of synthetic SARS-CoV-2 RNA. Bars represent 95\% Total Poisson Confidence Intervals. (B). Precision of each primer/probe set, defined as the coefficient of variation (expressed as a percentage, $\mathrm{CV} \%$ ) of measured copies, is shown for reactions containing 1,000 and 100 input copies of synthetic SARS-CoV-2 RNA. (C). Plotting precision versus analytical efficiency at 1,000 input SARS-CoV-2 RNA copies identifies E-Sarbeco, IP2 and IP4 primer/probe sets as having analytical efficiencies $>50 \%$ and $\mathrm{CV}(\%)<15 \%$. (D). Same as C, but for 100 input SARS-CoV-2 RNA copies.

Figure 3: Linear Dynamic Range (LDR) of E-Sarbeco, IP2 and IP4 RT-ddPCR assays. (A). left: $\log _{10}$ Measured SARS-CoV-2 RNA copies over serial dilutions of synthetic SARS-CoV-2 RNA standards ranging from 114,286 to 2.32 copies/reaction (shown as $\log _{10}$ values), using the 
E-Sarbeco primer/probe set. Error bars indicate 95\% Total Poisson Confidence Intervals for two merged replicates, where in some cases error bars are too small to visualize. The regression line joins all data points included in the LDR, where the lower boundary of the LDR represents the lower limit of quantification (LLOQ) of the assay. Data points that yielded undetectable measurements are set arbitrarily to $-0.35 \log _{10}$ Measured copies/reaction for visualization. right: $\log _{10}$ Residuals, calculated as $\log _{10}$ Measured SARS-CoV-2 RNA copies/reaction minus $\log _{10}$ Calulated SARS-CoV-2 RNA copies/reaction from the LDR regression. Grey shading indicates data points outside the LDR. Residuals for data points that yielded undetectable measurements are arbitrarily set to -0.4 for visualization. (B). Same as A, but for the IP2 primer/probe set (C). Same as A, but for the IP4 primer/probe set.

\section{Figure 4: Lower Limit of Detection (LLOD) of the E-Sarbeco, IP2 and IP4 RT-ddPCR}

assays. (A). The probability of detecting SARS-CoV-2 RNA (\%) in 1:2 in serial dilutions of synthetic SARS-CoV-2 RNA from 47.6 to 0.74 input copies/reaction using the E-Sarbeco primer/probe set is analyzed using probit regression (solid black line; dashed line denotes the 95\% confidence interval). The LLOD, defined as the concentration of SARS-CoV-2 RNA in a reaction where the probability of detection in the assay was $95 \%$, was interpolated from the standard curve and is shown as a colored dashed line (B). Same as A, but for the IP2 primer/probe set (C). Same as A, but for the IP4 primer/probe set.

Figure 5: $\log _{10}$ SARS-CoV-2 RNA loads in diagnostic specimens (A). SARS-CoV-2 E (green circles), ORF1a (red squares) and ORF1b (blue triangles) gene copy numbers, expressed as RNA copies/ $\mu 1$ of nucleic acid extract. Line and bars indicate median and interquartile range, 
respectively. (B) Correlation between $\log _{10}$ SARS-CoV-2 E and ORF1a gene RNA copies/ $\mu 1$ extract. (C). Correlation between $\log _{10} \mathrm{SARS}-\mathrm{CoV}-2 \mathrm{E}$ and ORF1b gene RNA copies/ $\mu$ l extract. (D) Correlation and Concordance between $\log _{10} \mathrm{SARS}-\mathrm{CoV}-2$ ORF1a and ORF1b gene RNA copies/ $\mu$ l extract.

Figure 6: Relationship between SARS-CoV-2 RNA copies equivalent and diagnostic test $C_{t}$ value. $\mathrm{C}_{\mathrm{t}}$ value, determined using the LightMix ${ }^{\circledR}$ 2019-nCoV real-time RT-PCR assay (E-gene target) is plotted against $\log _{10} \mathrm{SARS}-\mathrm{CoV}-2 \mathrm{E}$ gene RNA copies equivalent, which represents the number of SARS-CoV-2 RNA copies measured by RT-ddPCR in $9 \mu 1$ extract (the template volume in the LightMix ${ }^{\circledR}$ assay). The linear regression (solid black line) transitions to a dashed line below the LLOQ.

\section{Supplementary Figure 1: All experiments using synthetic SARS-CoV-2 synthetic standards were performed in a consistent background of human nucleic acids to mimic a real human}

sample. Example experiment showing consistent levels of background human cells/ $\mu$ l extract (determined by dividing measured human RPP30 DNA copy number by two; black triangles), and human RNAse P RNA levels (grey squares) across a titration of SARS-CoV-2 synthetic RNA standards, measured using the E-Sarbeco primer/probe set (green circles). Error bars indicate 95\% Total Poisson Confidence Intervals for two merged replicates, where in some cases error bars are too small to visualize. Grey (RNase P) and black (RPP30) dashed lines indicate copies measured control experiments lacking SARS-CoV-2 RNA. 


\section{Supplementary Figure 2: Duplexing the IP2 and IP4 primer/probe sets reduces analytical} efficiency and precision. (A). Analytical efficiency of SARS-CoV-2 quantification was evaluated for the IP2 and IP4 primer/probe sets when used in separate reactions (dark red and dark blue, respectively) and when duplexed (light red and light blue, respectively), in reactions containing 1,000 and 100 viral RNA input copies. Error bars represent 95\% Total Poisson Confidence Intervals. (B). Same as A, but for assay precision (coefficient of variation, CV\%).

\section{Supplementary Figure 3: $\log _{10}$ SARS-CoV-2 RNA loads in diagnostic specimens,} normalized to human cells sampled. (A) SARS-CoV-2 E (green circles), ORF1a (red squares) and ORF1b (blue triangles) gene copy numbers, expressed as RNA copies/1,000 human cells. Line and bars indicate median and interquartile range, respectively. (B) Correlation between SARS-CoV-2 RNA copies/ $\mu$ l extract and RNA copies/1,000 human cells.

\section{Supplemental Figure 4: Residuals of relationship between SARS-CoV-2 RNA copies} equivalent and diagnostic test $\mathbf{C}_{\mathbf{t}}$ value. $\log _{10}$ Residuals are calculated as $\log _{10}$ Measured SARS-CoV-2 RNA copies equivalent minus $\log _{10}$ Calulated SARS-CoV-2 RNA copies equivalent from the regression line shown in Figure 6.

\section{Supplemental Figure 5: Relationship between SARS-CoV-2 RNA copies/1,000 human cells} and $\mathbf{C}_{\mathbf{t}}$ value. Same data as shown in Figure 6 , but where the measured SARS-CoV-2 RNA copies/ $\mu$ l extract were normalized to copies/1,000 human cells. The linear regression is shown as a solid black line. 


\section{E-Sarbeco Annealing/Extension}

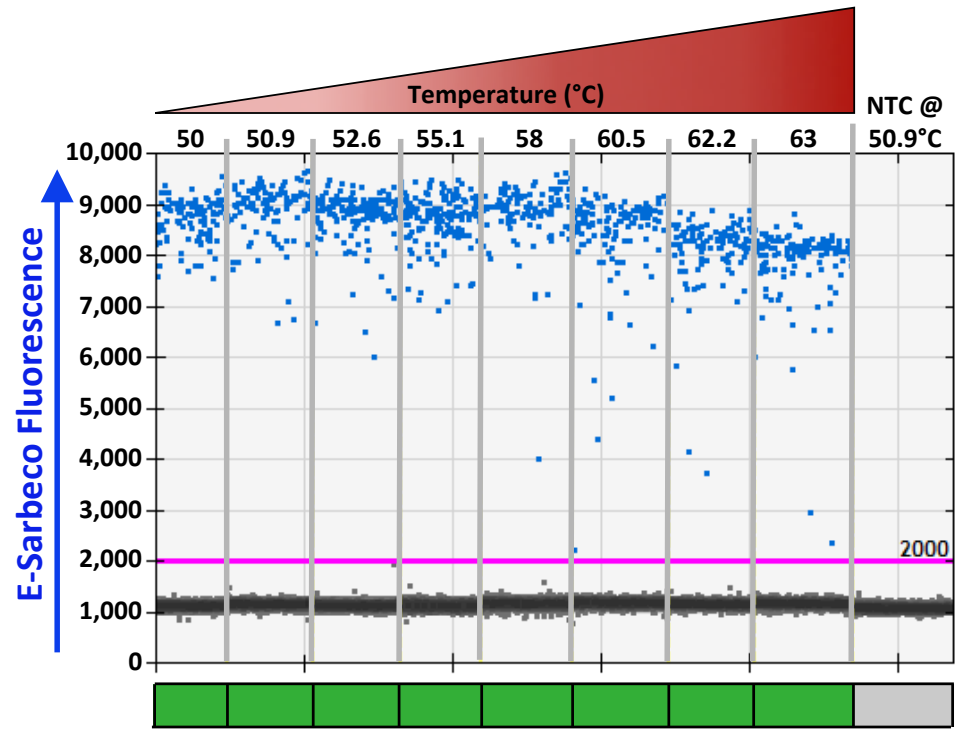

HKU-ORF Annealing/Extension

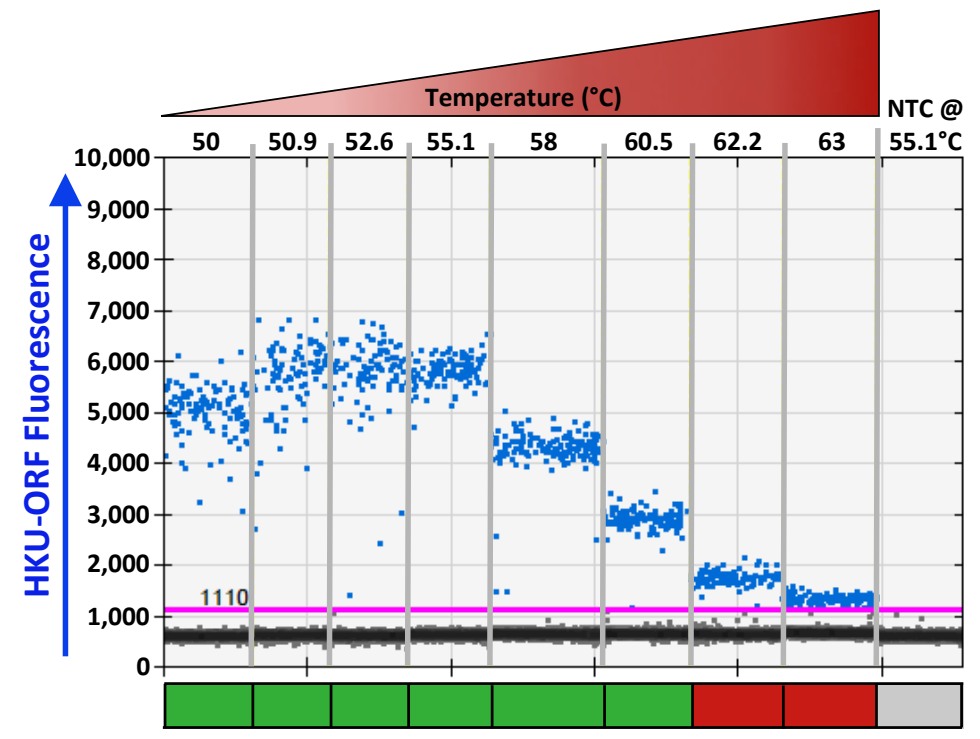

C

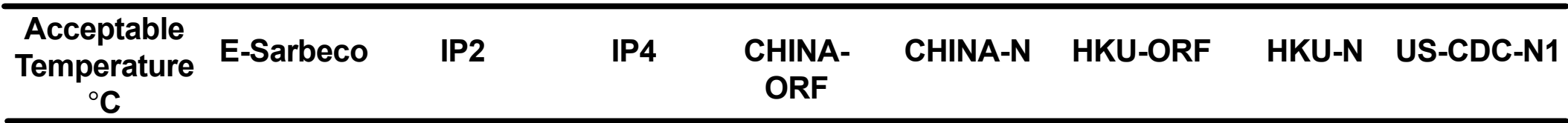

\begin{tabular}{ccccccccc}
\hline RT & $42-49.7$ & $42-51.5$ & $42-50.9$ & $42-51.5$ & $42.7-50.9$ & $42-51.5$ & $42-51.5$ & $42-45.7$ \\
\hline $\begin{array}{l}\text { Annealing/ } \\
\text { Extension }\end{array}$ & $50-63$ & $50-60.5$ & $50-60.5$ & $50-63$ & $50-60.5$ & $50-60.5$ & $50.9-60.5$ & $50-63$ \\
\hline
\end{tabular}

Figure 1: Thermal cycling optimization (A). RT-ddPCR plots for annealing/extension under a $50-63^{\circ} \mathrm{C}$ thermal gradient for the E-Sarbeco primer/probe set. A representative RT-ddPCR plot for a no template control (NTC) which only included non-target DNA/RNA (see methods) at the temperature used in subsequent experiments, is also shown. Positive droplets (blue) are above the threshold (pink line); negative droplets (grey) are below the line. Colored boxes below each well indicate if results met standards for inclusion (green) or not (red) (see methods). (B). Same as panel A, but for HKU-ORF primer/probe set. (C). Acceptable RT and annealing/extension temperature ranges for each primer/probe set. 

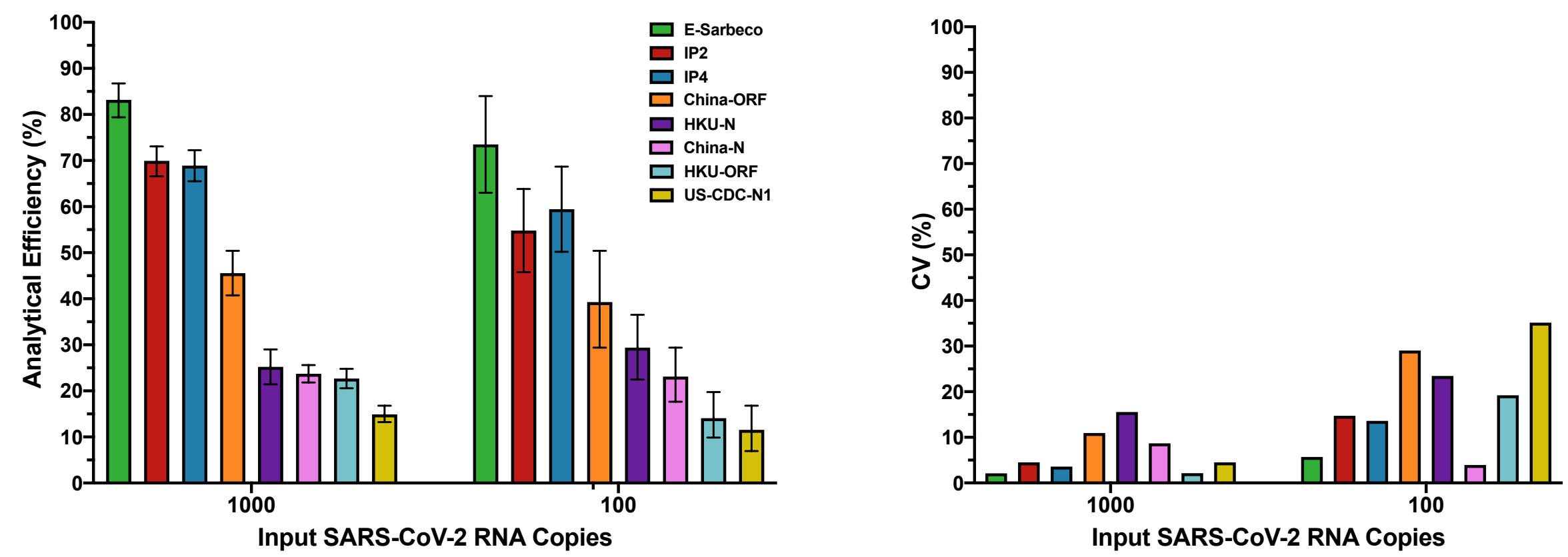

C

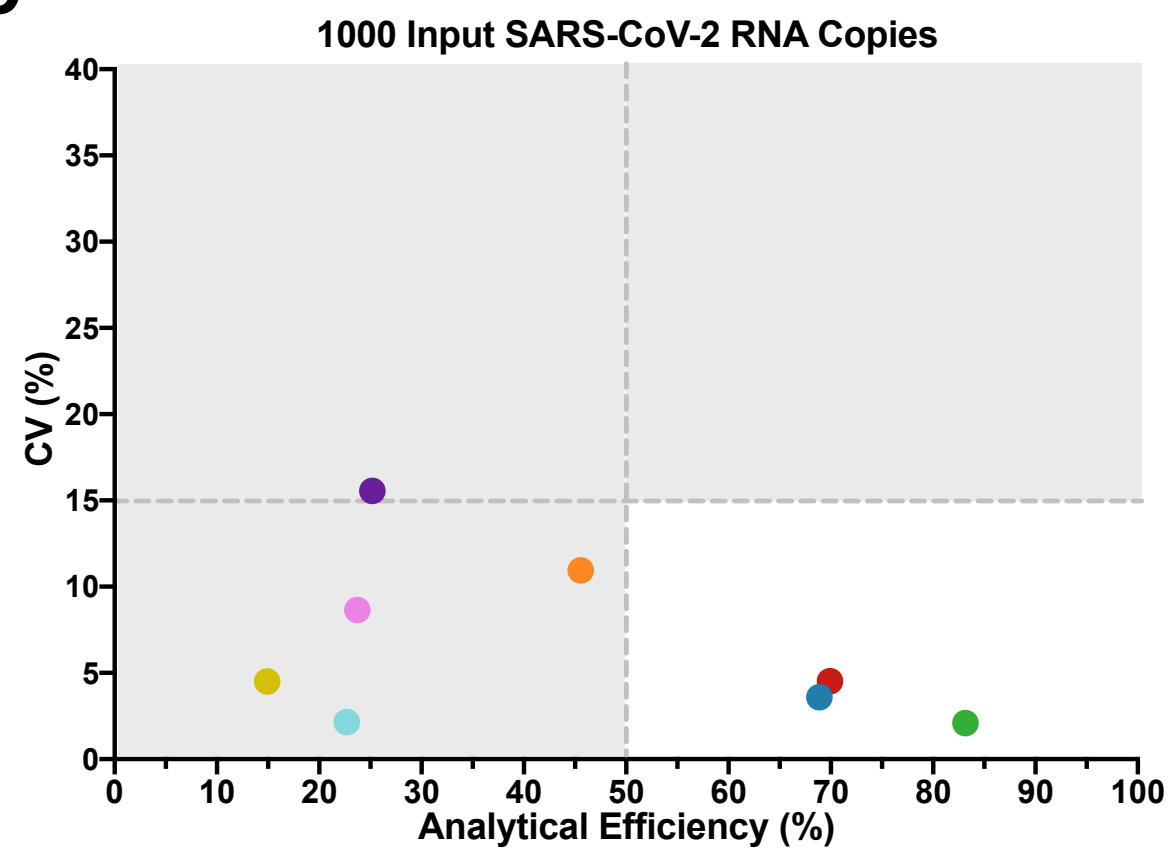

D

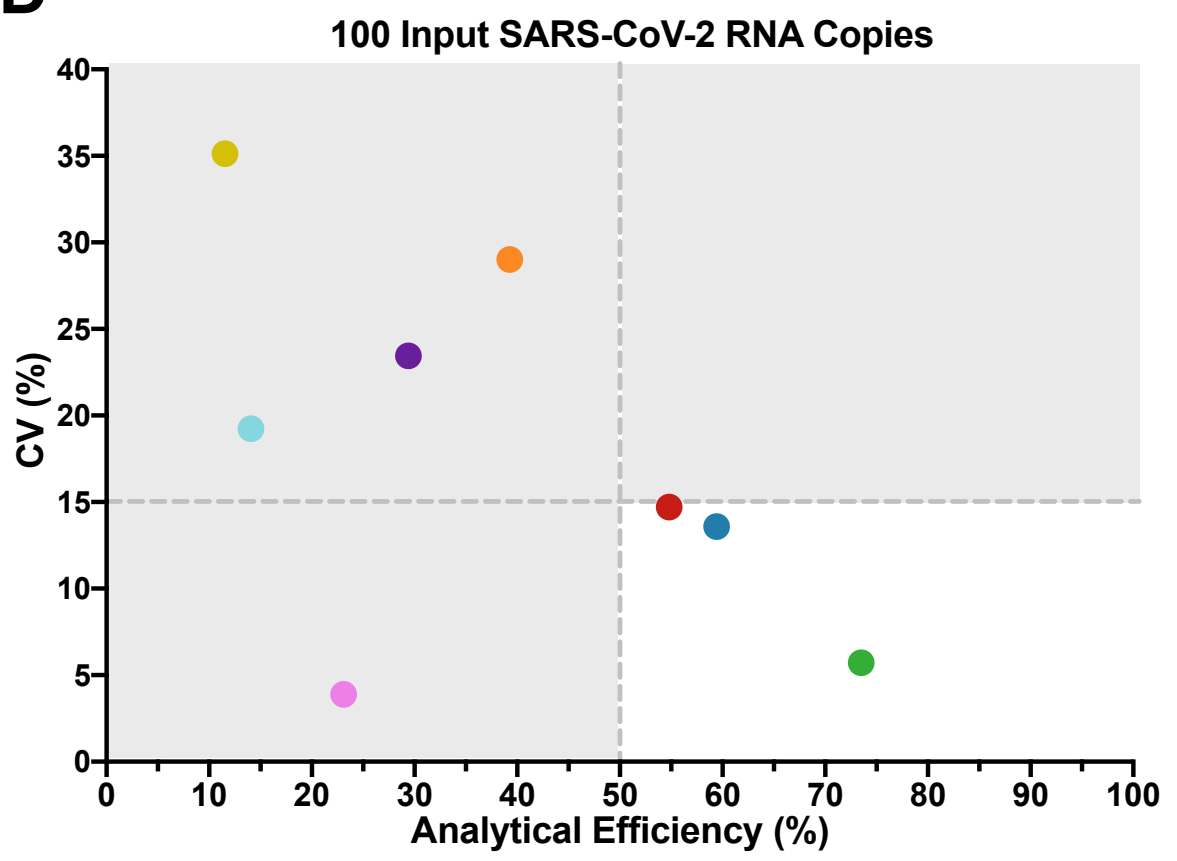


Figure 2: Analytical efficiency and precision of primer/probe sets. (A) Analytical efficiency of each primer/probe set, calculated as the measured divided by the input SARS-CoV-2 RNA copies multiplied by $100 \%$, is shown for reactions containing 1,000 and 100 input copies of synthetic SARS-CoV-2 RNA. Bars represent 95\% Total Poisson Confidence Intervals. (B). Precision of each primer/probe set, defined as the coefficient of variation (expressed as a percentage, $\mathrm{CV} \%$ ) of measured copies, is shown for reactions containing 1,000 and 100 input copies of synthetic SARS-CoV-2 RNA. (C). Plotting precision versus analytical efficiency at 1,000 input SARS-CoV-2 RNA copies identifies E-Sarbeco, IP2 and IP4 primer/probe sets as having analytical efficiencies $>50 \%$ and CV $(\%)<15 \%$. (D). Same as C, but for 100 input SARS-CoV-2 RNA copies. 
$\mathbf{A}$

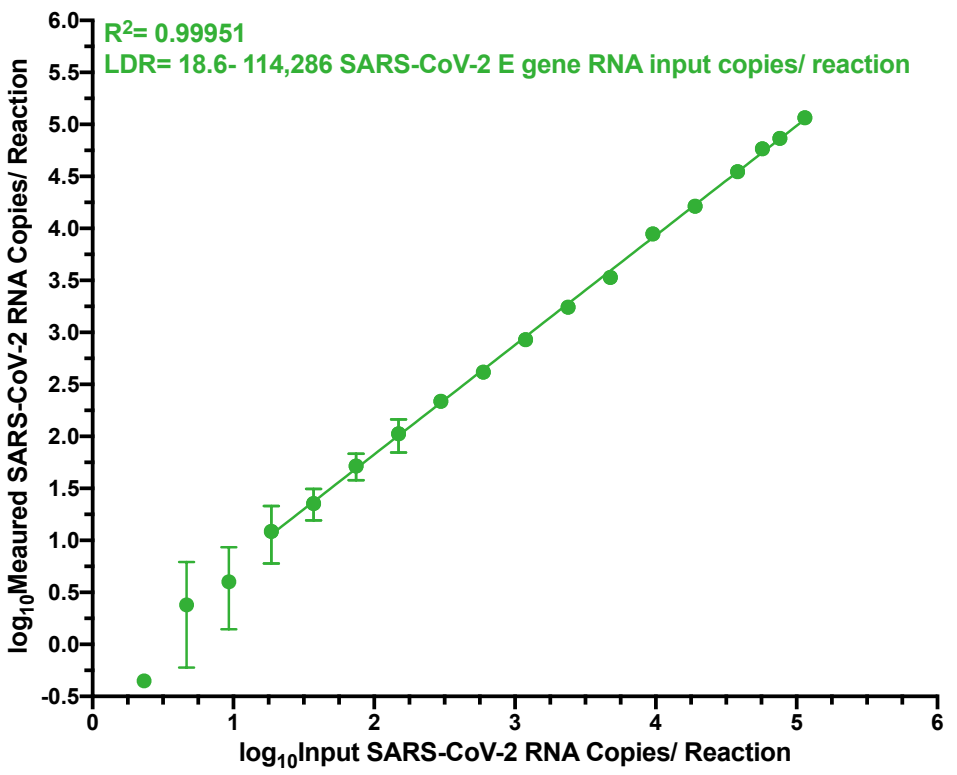

B

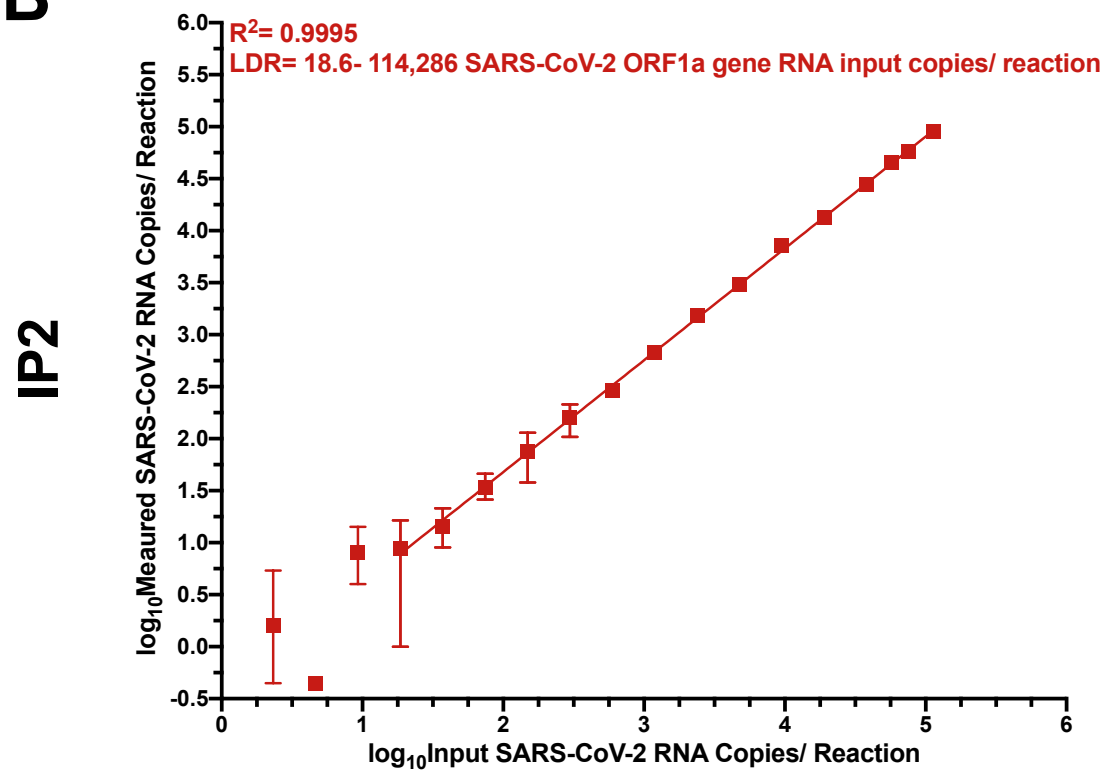

$\underline{\mathbf{N}}$

C

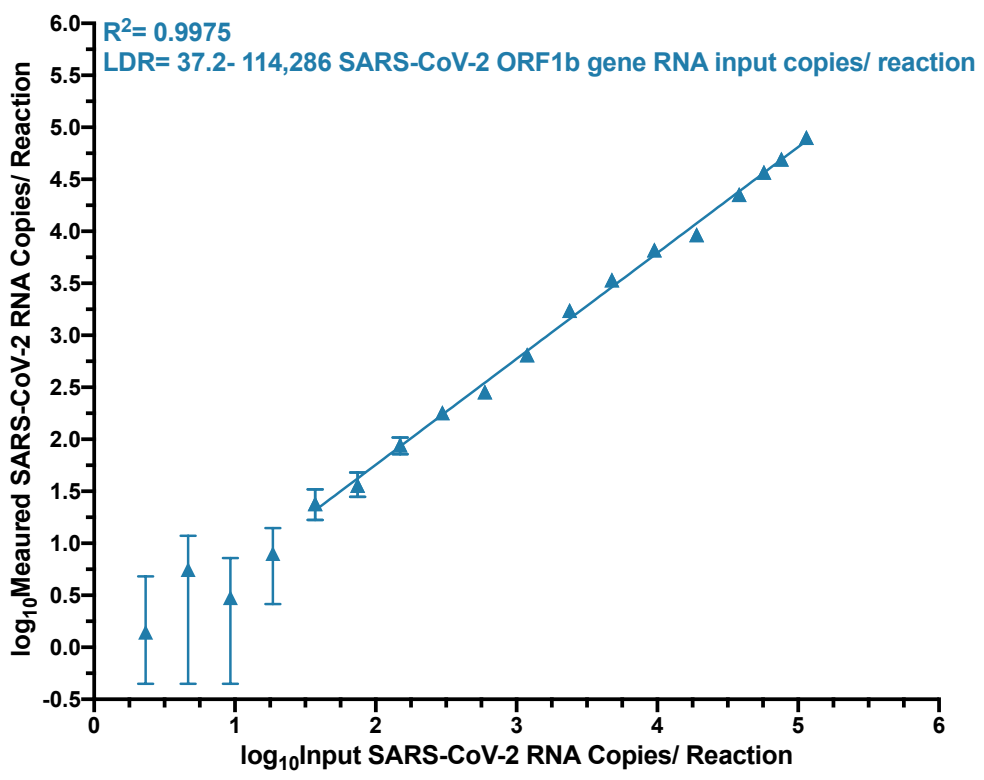

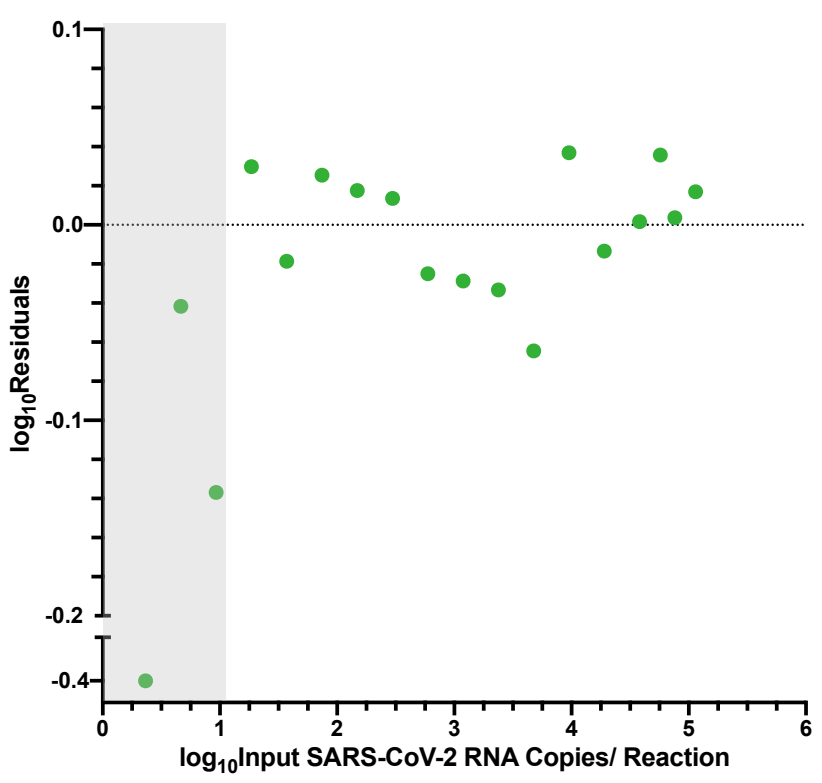
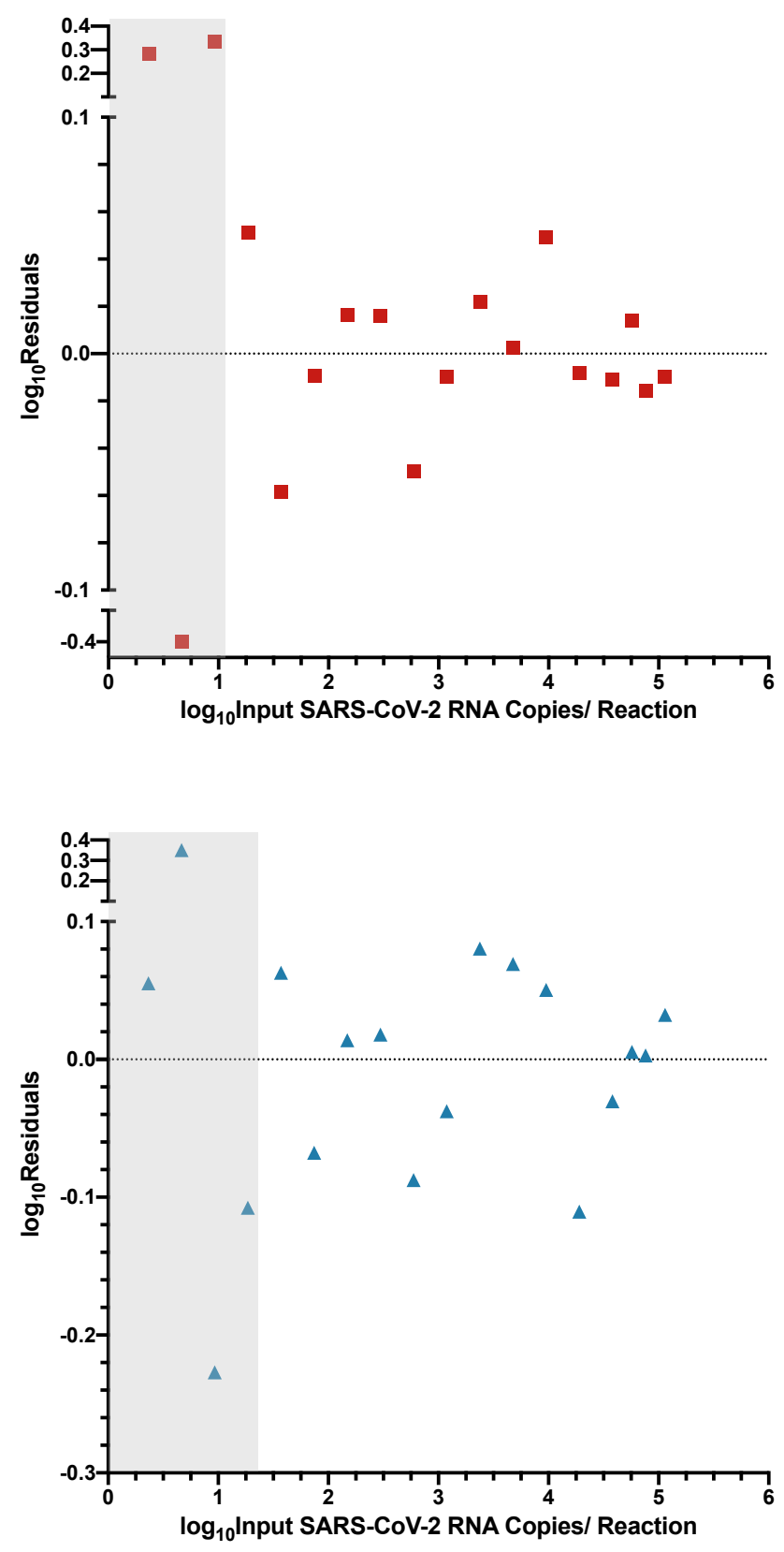
Figure 3: Linear Dynamic Range (LDR) of E-Sarbeco, IP2 and IP4 RT-ddPCR assays. (A). left: $\log _{10}$ Measured SARS-CoV-2 RNA copies over serial dilutions of synthetic SARS-CoV-2 RNA standards ranging from 114,286 to 2.32 copies/reaction (shown as $\log _{10}$ values), using the E-Sarbeco primer/probe set. Error bars indicate 95\% Total Poisson Confidence Intervals for two merged replicates, where in some cases error bars are too small to visualize. The regression line joins all data points included in the LDR, where the lower boundary of the LDR represents the lower limit of quantification (LLOQ) of the assay. Data points that yielded undetectable measurements are set arbitrarily to $-0.35 \log _{10}$ Measured copies/reaction for visualization. right: $\log _{10}$ Residuals, calculated as $\log _{10}$ Measured SARS-CoV-2 RNA copies/reaction minus $\log _{10}$ Calulated SARS-CoV-2 RNA copies/reaction from the LDR regression. Grey shading indicates data points outside the LDR. Residuals for data points that yielded undetectable measurements are arbitrarily set to -0.4 for visualization. (B). Same as A, but for the IP2 primer/probe set (C). Same as A, but for the IP4 primer/probe set. 


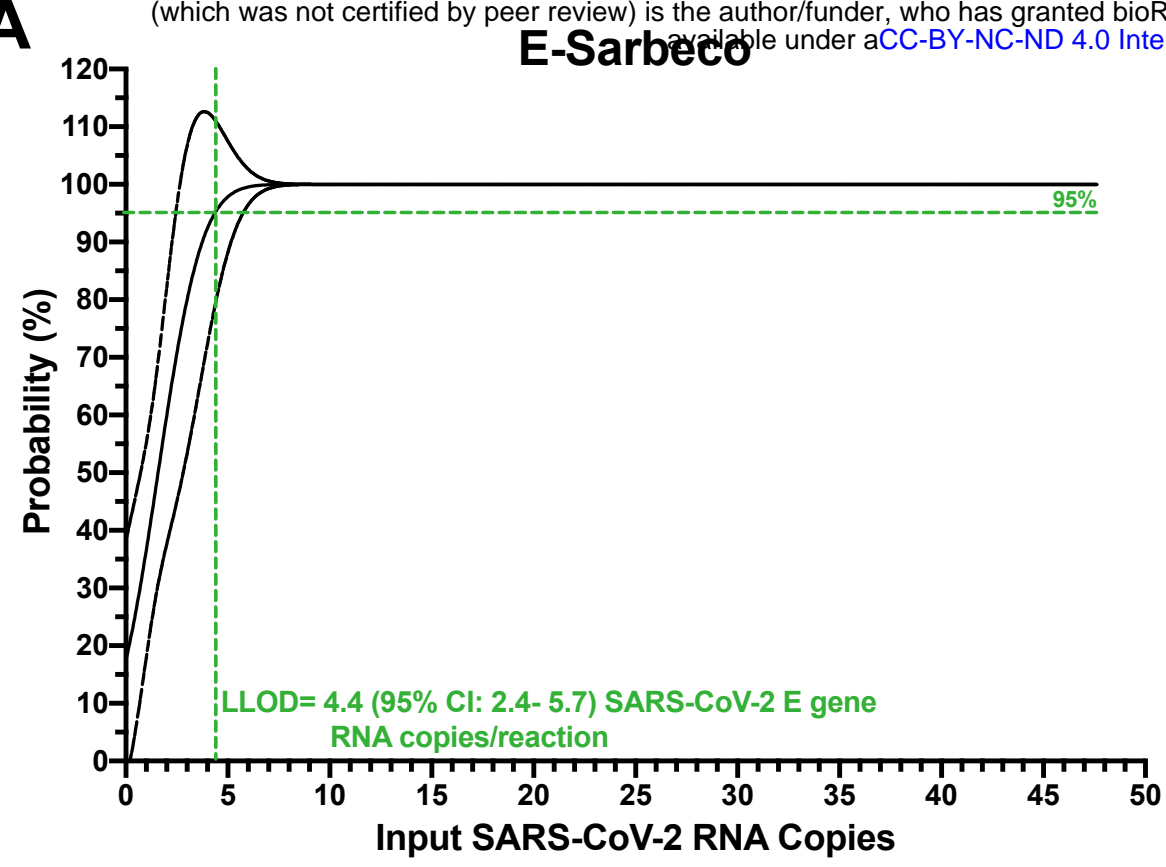

B

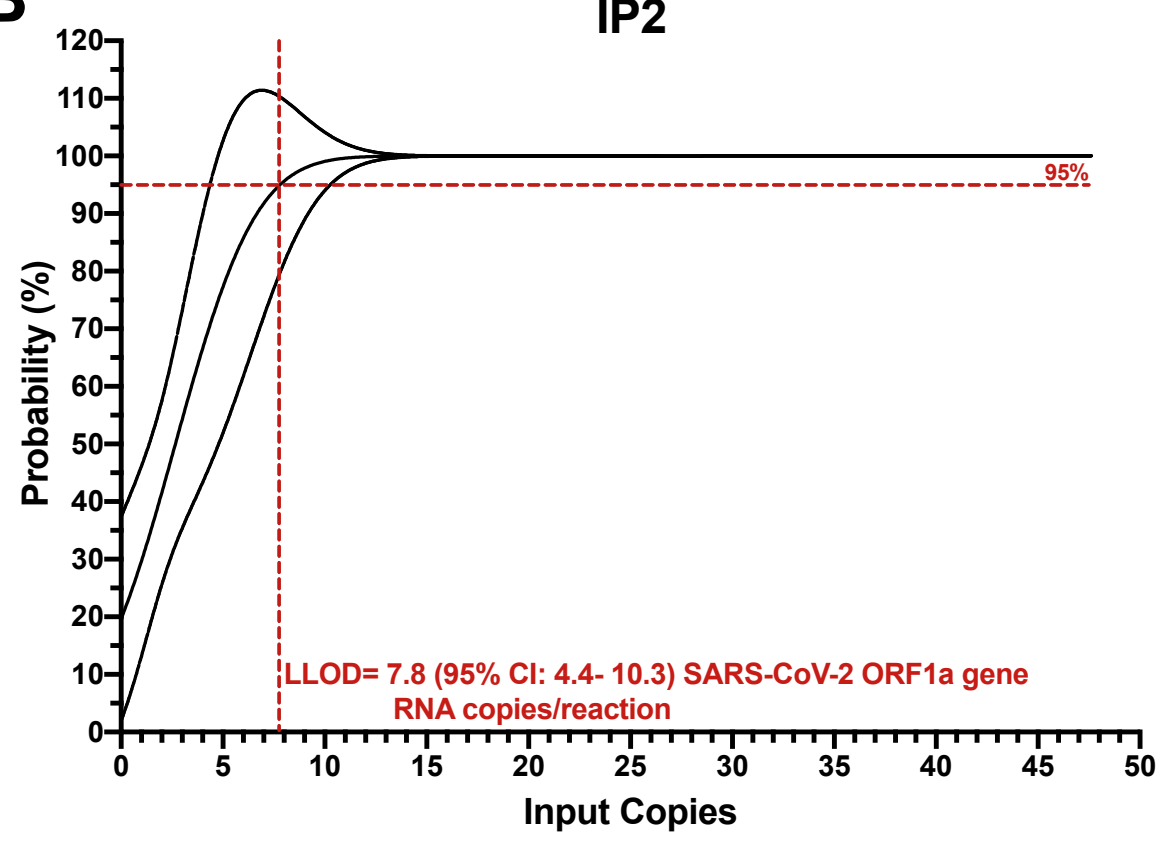

C

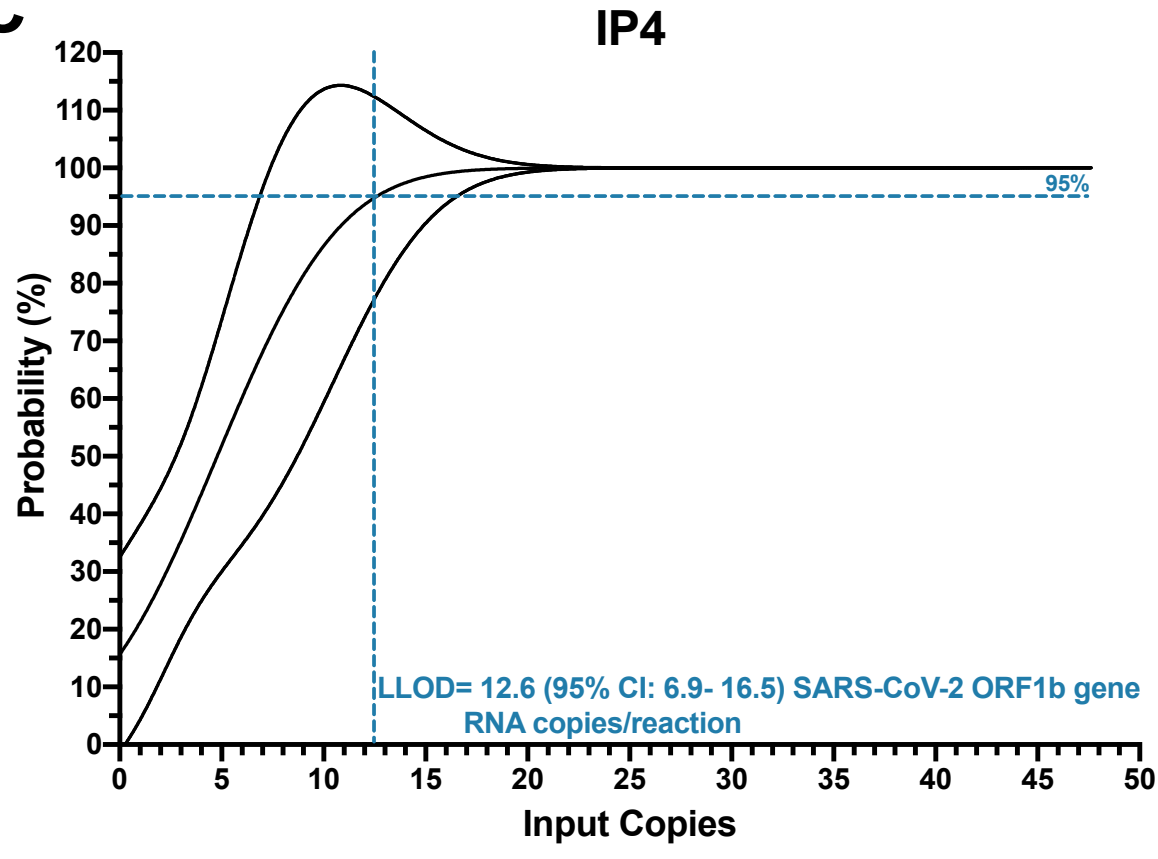


Figure 4: Lower Limit of Detection (LLOD) of the E-Sarbeco, IP2 and IP4 RTddPCR assays. (A). The probability of detecting SARS-CoV-2 RNA (\%) in 1:2 in serial dilutions of synthetic SARS-CoV-2 RNA from 47.6 to 0.74 input copies/reaction using the E-Sarbeco primer/probe set is analyzed using probit regression (solid black line; dashed line denotes the $95 \%$ confidence interval). The LLOD, defined as the concentration of SARS-CoV-2 RNA in a reaction where the probability of detection in the assay was $95 \%$, was interpolated from the standard curve and is shown as a colored dashed line (B). Same as A, but for the IP2 primer/probe set (C). Same as A, but for the IP4 primer/probe set. 
A

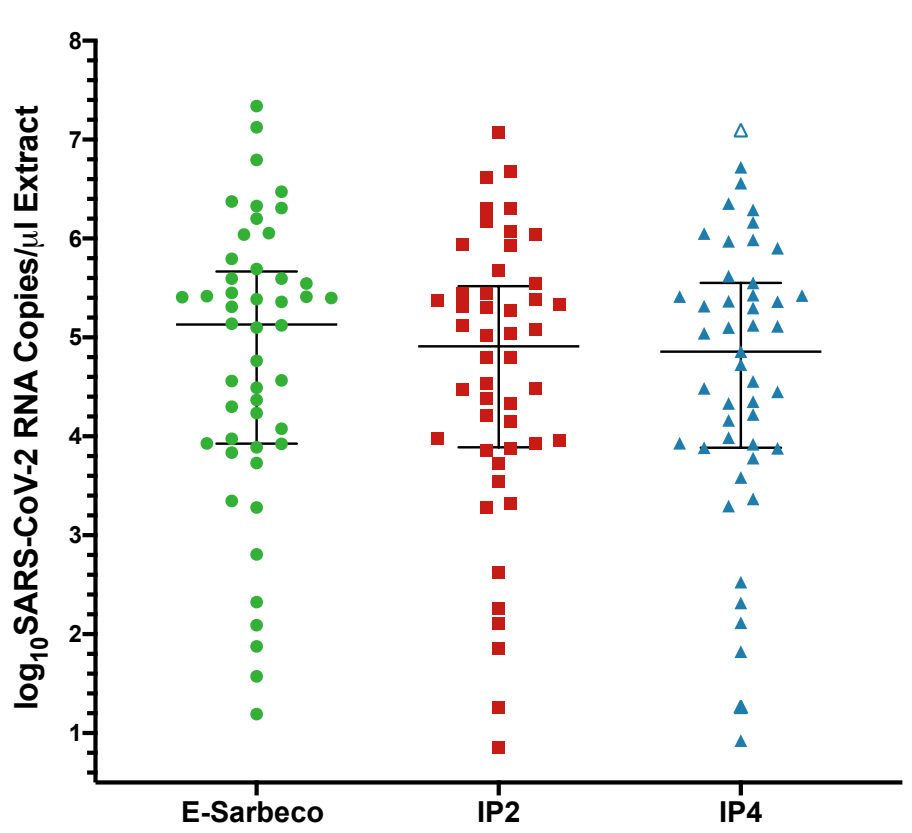

C

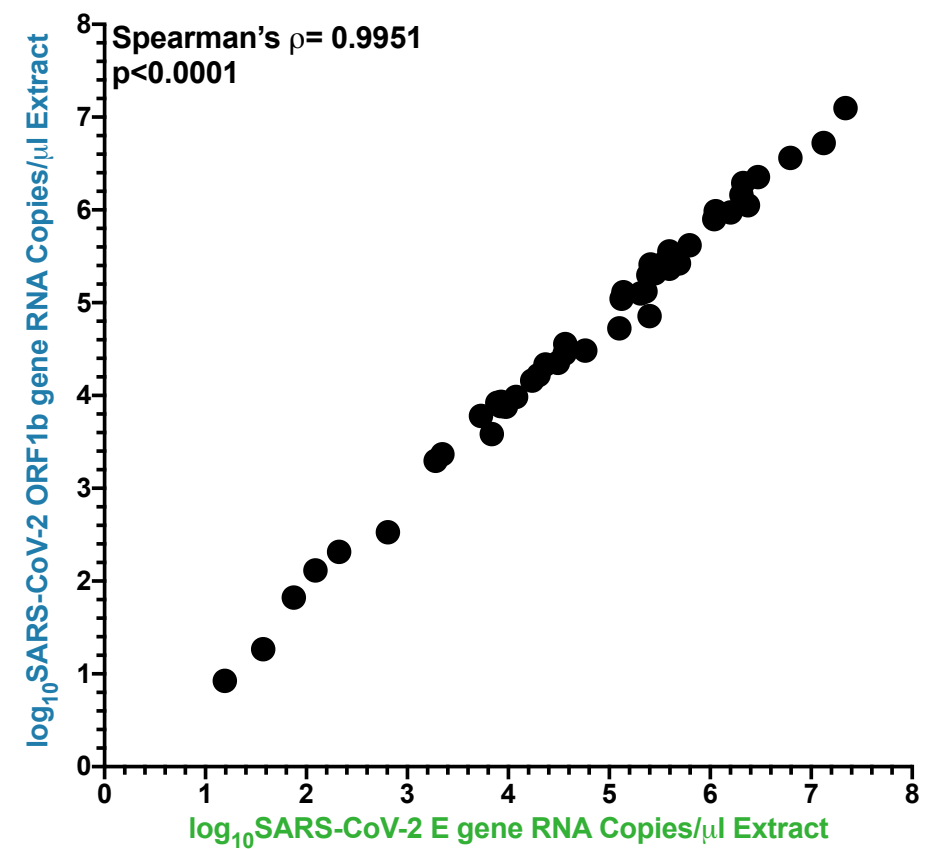

B

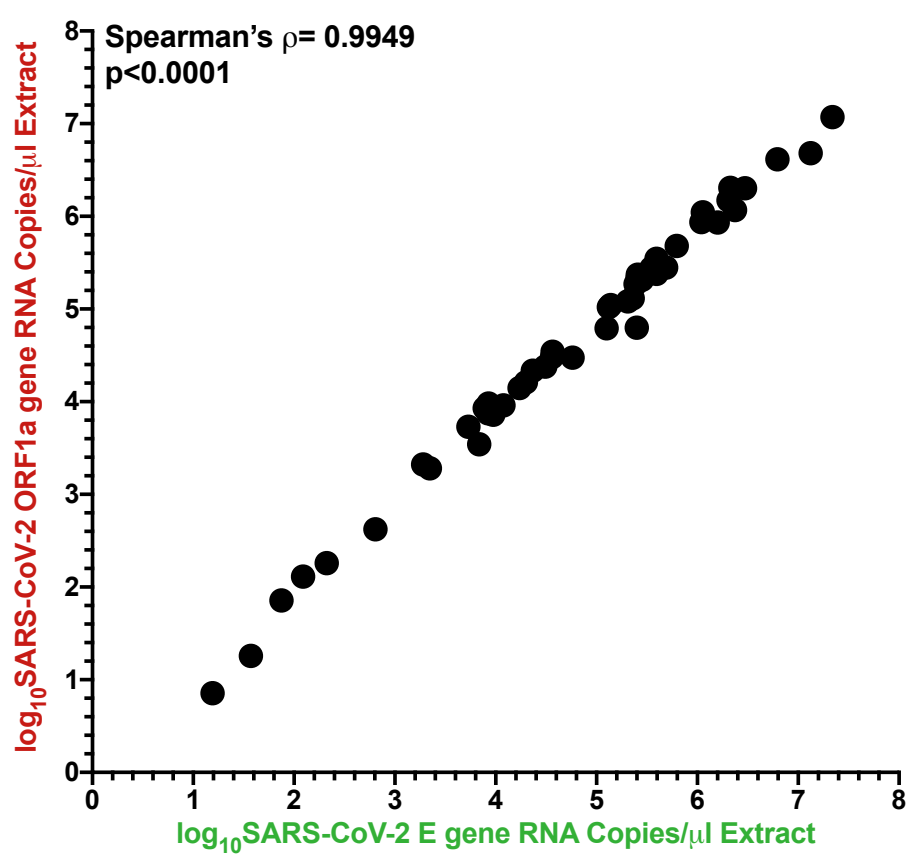

D

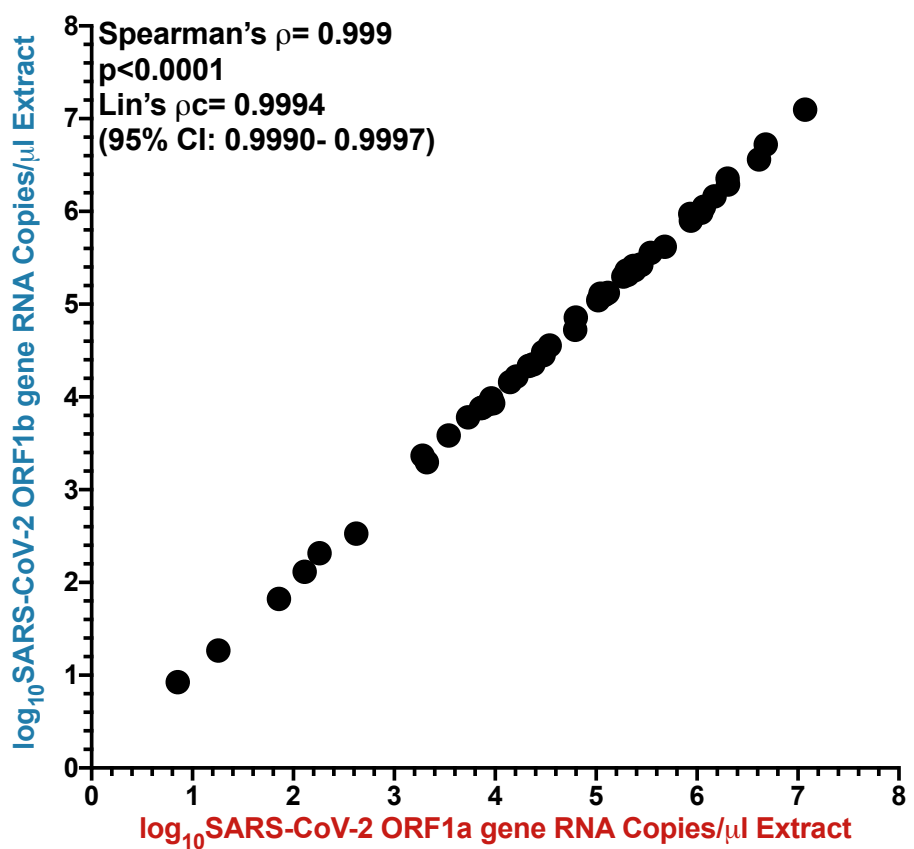


Figure 5: $\log _{10}$ SARS-CoV-2 RNA loads in diagnostic specimens (A). SARS-CoV-2 E (green circles), ORF1a (red squares) and ORF1b (blue triangles) gene copy numbers, expressed as RNA copies/ $\mu$ l of nucleic acid extract. Line and bars indicate median and interquartile range, respectively. (B) Correlation between $\log _{10}$ SARS-CoV-2 E and

ORF1a gene RNA copies/ $\mu$ l extract. (C). Correlation between $\log _{10} \mathrm{SARS}-\mathrm{CoV}-2 \mathrm{E}$ and ORF1b gene RNA copies/ $\mu$ l extract. (D) Correlation and Concordance between $\log _{10}$ SARS-CoV-2 ORF1a and ORF1b gene RNA copies/ $\mu 1$ extract. 


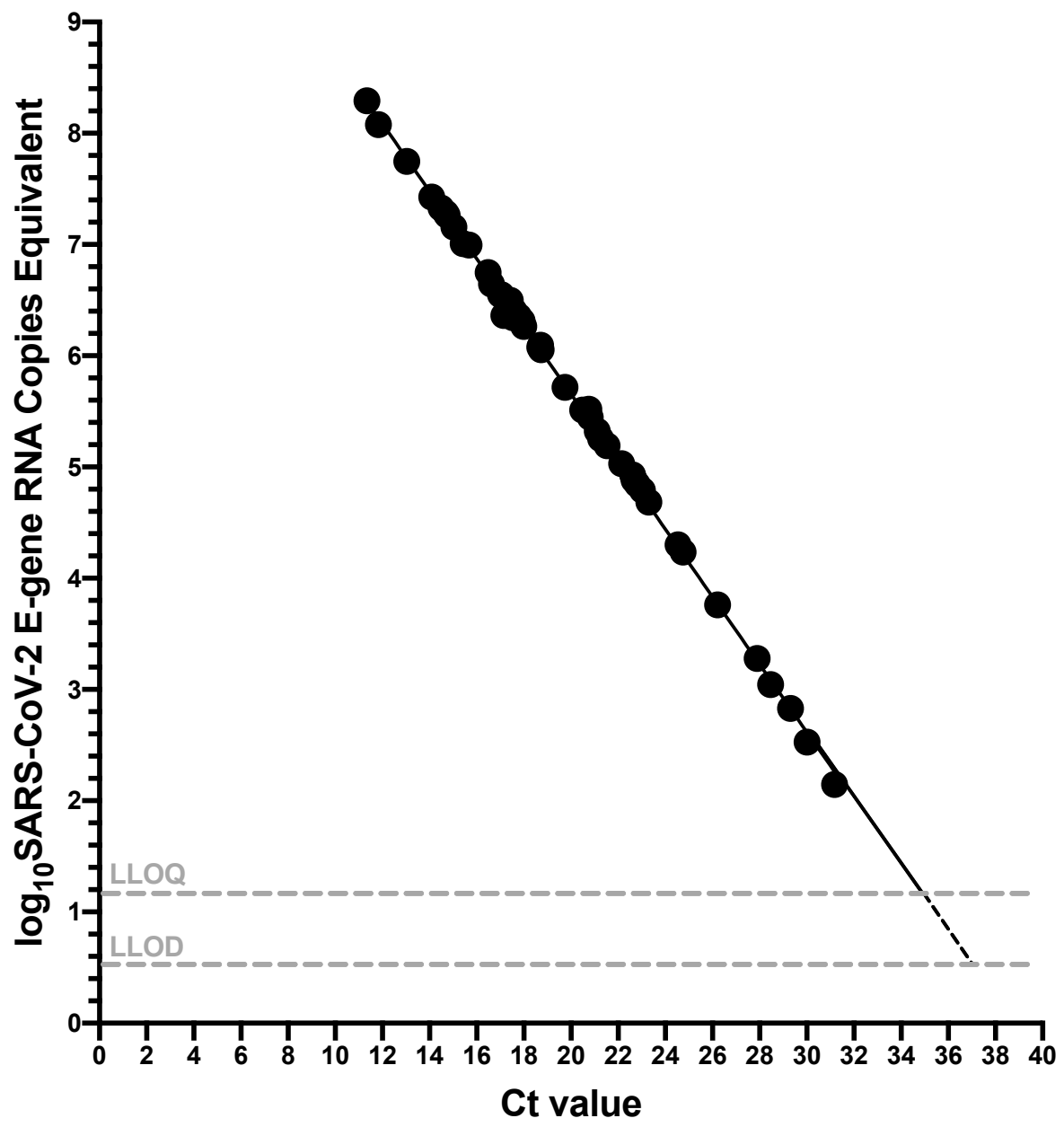

$R^{2}=0.99901$ $\log _{10}$ SARS-CoV-2 E-gene RNA Copies Equivalent $=-0.3038\left(C_{t}\right)+11.7$ 
Figure 6: Relationship between SARS-CoV-2 RNA copies equivalent and diagnostic test $\mathbf{C}_{t}$ value. $C_{t}$ value, determined using the LightMix ${ }^{\circledR}$ 2019-nCoV real-time RT-PCR assay (E-gene target) is plotted against $\log _{10}$ SARS-CoV-2 E gene RNA copies equivalent, which represents the number of SARS-CoV-2 RNA copies measured by RTddPCR in $9 \mu$ l extract (the template volume in the LightMix ${ }^{\circledR}$ assay). The linear regression (solid black line) transitions to a dashed line below the LLOQ. 


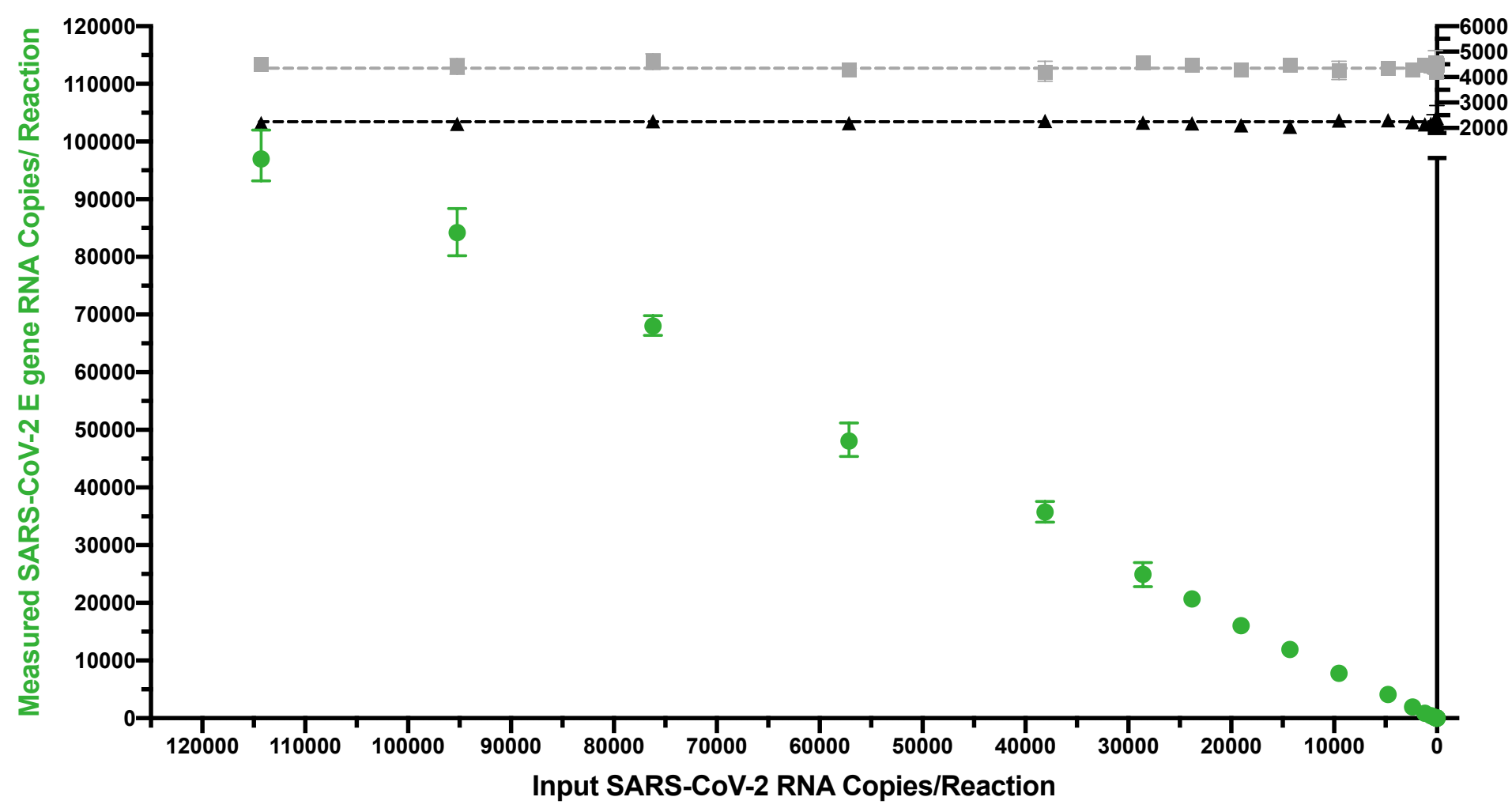


Supplementary Figure 1: All experiments using synthetic SARS-CoV-2 synthetic standards were performed in a consistent background of human nucleic acids to mimic a real human sample. Example experiment showing consistent levels of background human cells/ $\mu$ l extract (determined by dividing measured human RPP30 DNA copy number by two; black triangles), and human RNAse P RNA levels (grey squares) across a titration of SARS-CoV-2 synthetic RNA standards, measured using the E-Sarbeco primer/probe set (green circles). Error bars indicate 95\% Total Poisson

Confidence Intervals for two merged replicates, where in some cases error bars are too small to visualize. Grey (RNase P) and black (RPP30) dashed lines indicate copies measured control experiments lacking SARS-CoV-2 RNA. 


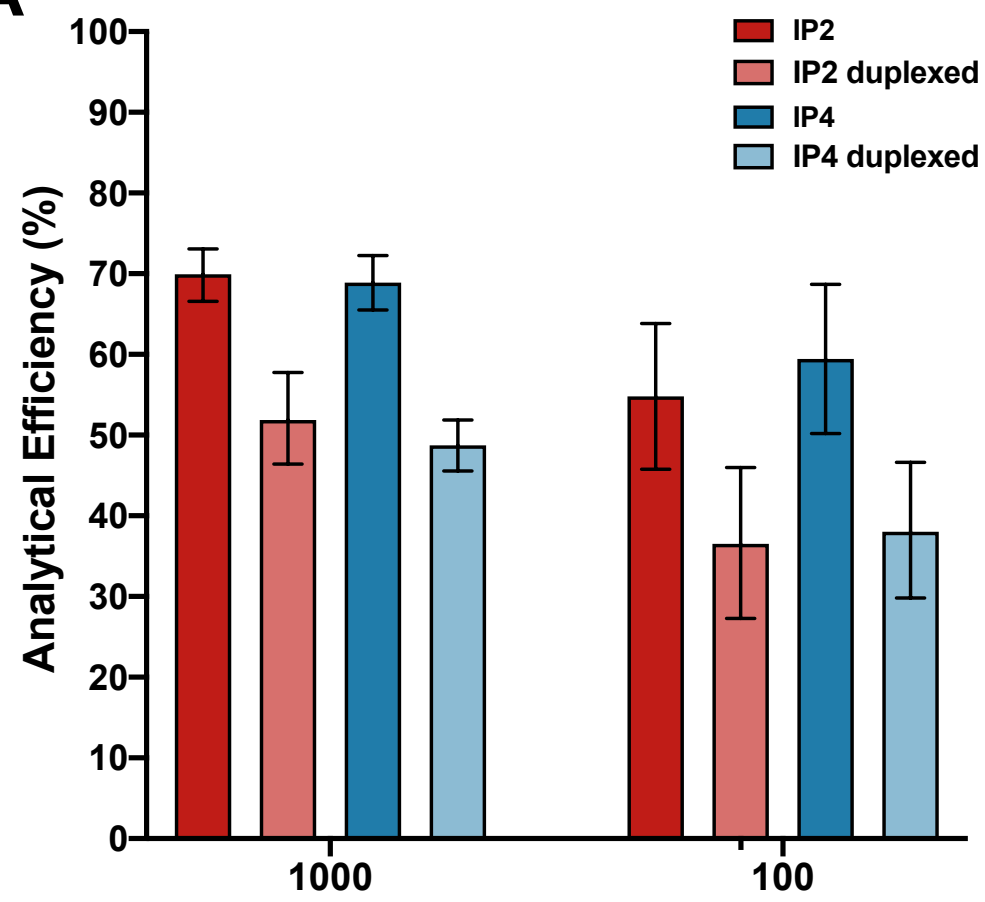
Input SARS-CoV-2 RNA Copies
B

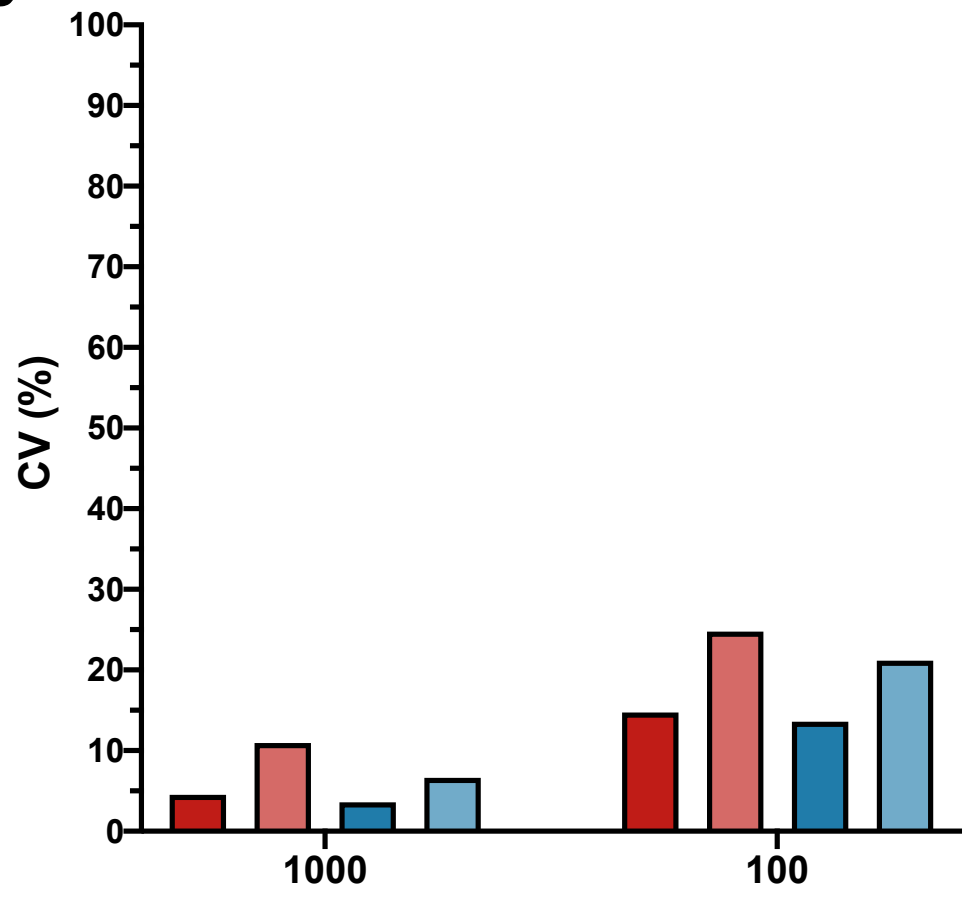
Input SARS-CoV-2 RNA Copies 
Supplementary Figure 2: Duplexing the IP2 and IP4 primer/probe sets reduces analytical efficiency and precision. (A). Analytical efficiency of SARS-CoV-2

quantification was evaluated for the IP2 and IP4 primer/probe sets when used in separate reactions (dark red and dark blue, respectively) and when duplexed (light red and light blue, respectively), in reactions containing 1,000 and 100 viral RNA input copies. Error bars represent 95\% Total Poisson Confidence Intervals. (B). Same as A, but for assay precision (coefficient of variation, $\mathrm{CV} \%$ ). 

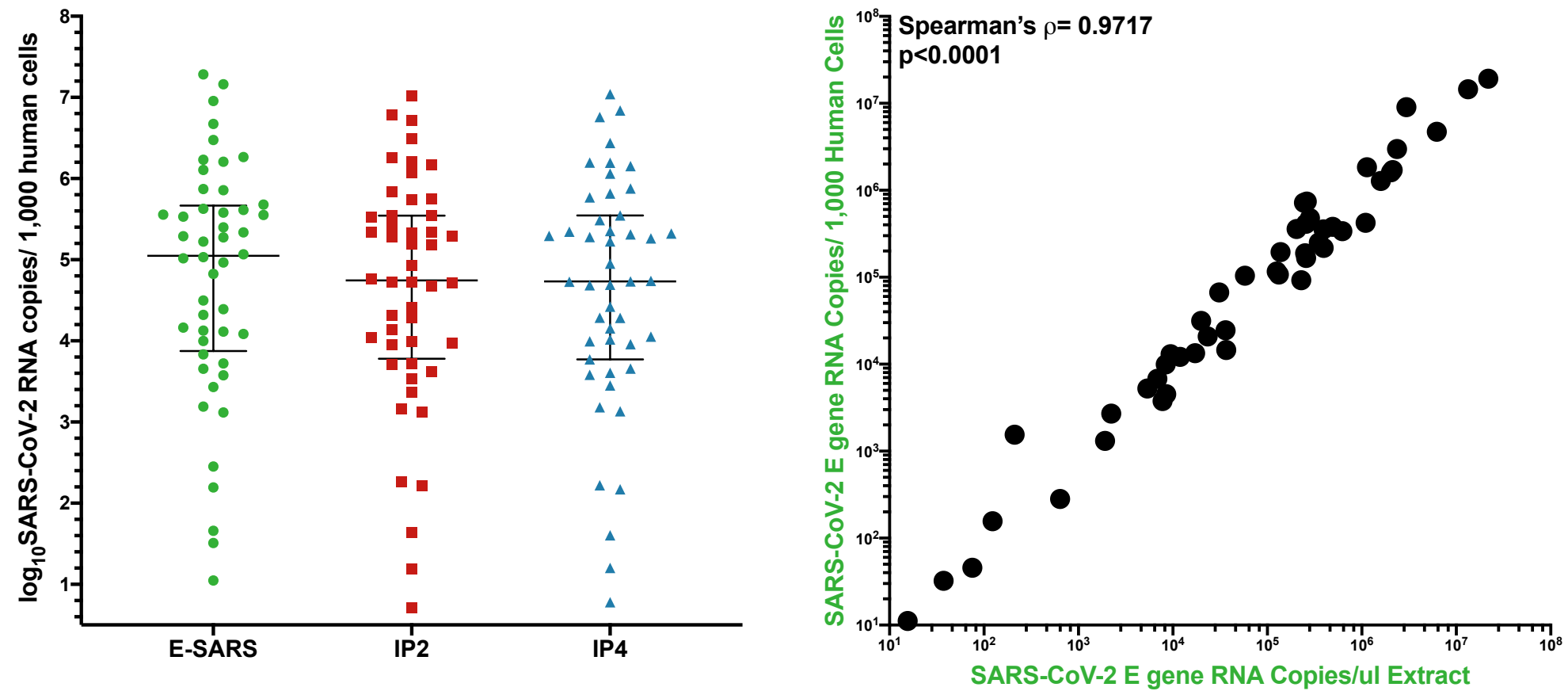
Supplementary Figure 3: $\log _{10}$ SARS-CoV-2 RNA loads in diagnostic specimens, normalized to human cells sampled. (A) SARS-CoV-2 E (green circles), ORF1a (red squares) and ORF1b (blue triangles) gene copy numbers, expressed as RNA copies/1,000 human cells. Line and bars indicate median and interquartile range, respectively. (B) Correlation between SARS-CoV-2 RNA copies/ $\mu$ l extract and RNA copies/1,000 human cells. 


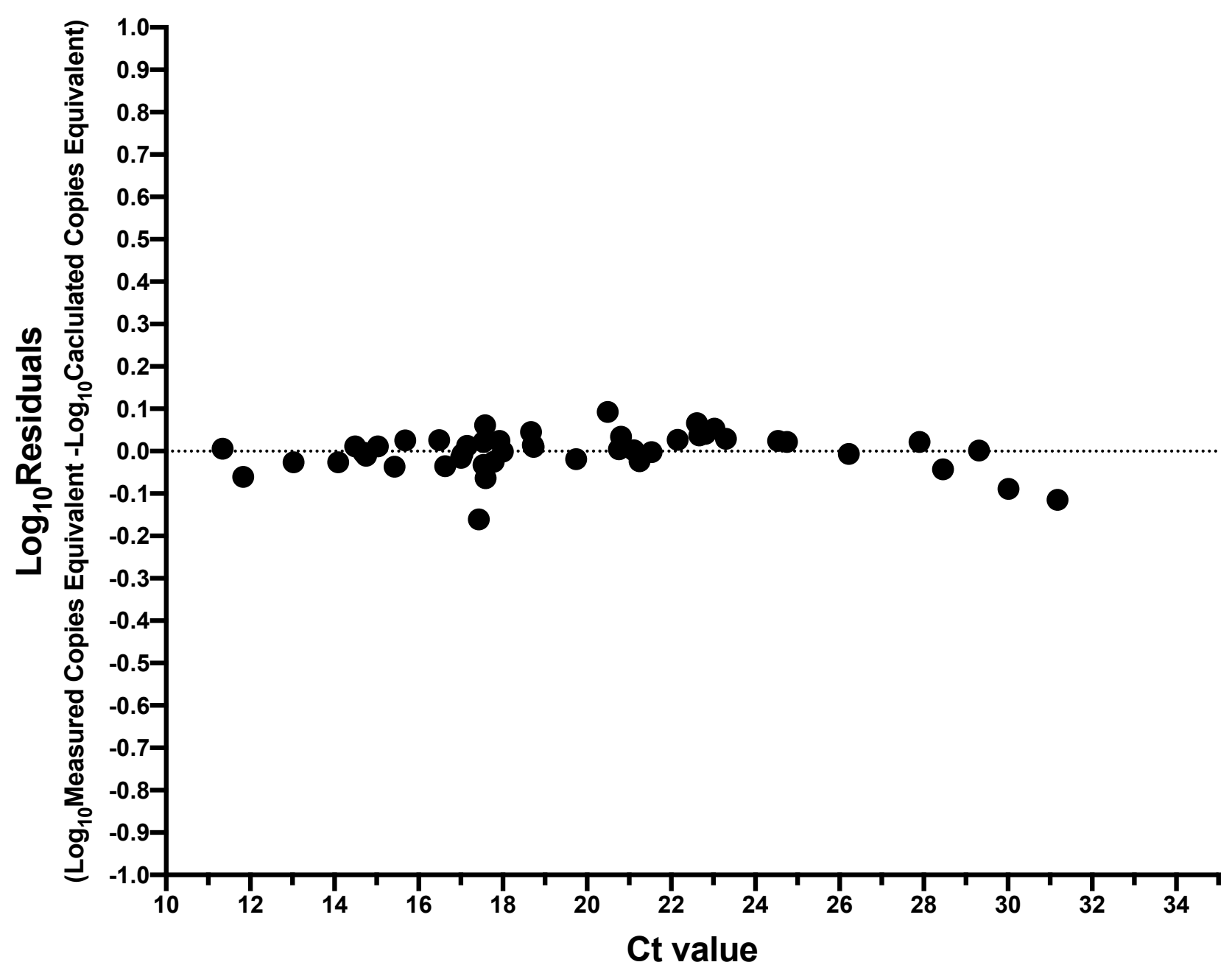


Supplemental Figure 4: Residuals of relationship between SARS-CoV-2 RNA copies equivalent and diagnostic test $\mathbf{C}_{\mathbf{t}}$ value. $\log _{10}$ Residuals are calculated as $\log _{10}$ Measured SARS-CoV-2 RNA copies equivalent minus $\log _{10}$ Calulated SARS-CoV-2 RNA copies equivalent from the regression line shown in Figure 6. 


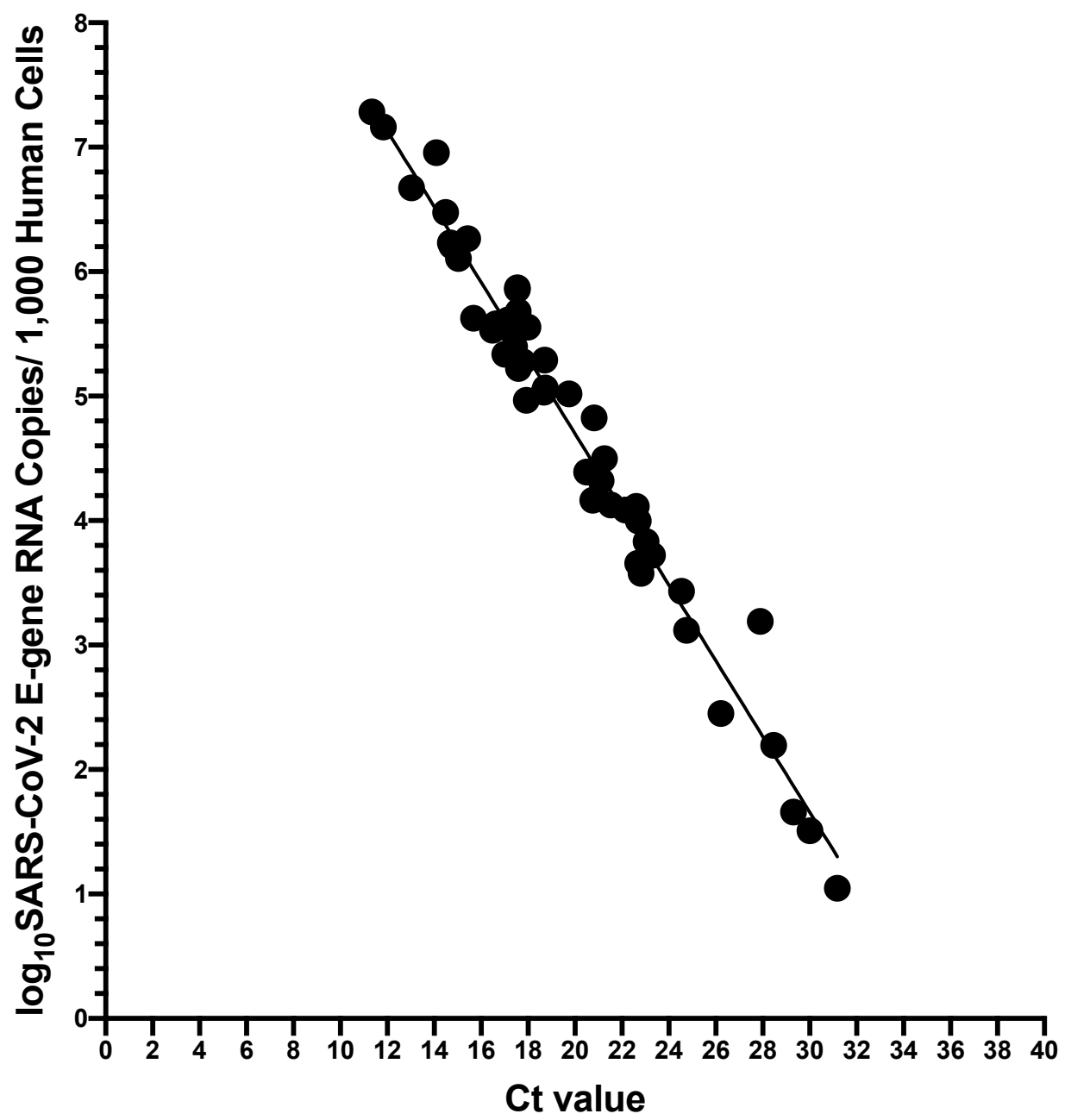

$R^{2}=0.97079$

$\log _{10}$ SARS-CoV-2 E-gene RNA Copies/1,000 Human Cells $=-0.3041\left(C_{t}\right)+10.8$ 
Supplemental Figure 5: Relationship between SARS-CoV-2 RNA copies/1,000 human cells and $\mathbf{C}_{\mathbf{t}}$ value. Same data as shown in Figure 6, but where the measured SARS-CoV-2 RNA copies/ $\mu$ l extract were normalized to copies/1,000 human cells. The linear regression is shown as a solid black line. 